\title{
Molecular Layer Deposition of Crosslinked Polymeric Lithicone for Superior Lithium Metal Anodes
}

\author{
Xiangbo Meng $\mathbb{D}^{1},{ }^{1}$ Kah Chun Lau $\mathbb{D}^{2}{ }^{2}$ Hua Zhou, ${ }^{3}$ Sujan Kumar Ghosh, ${ }^{1}$ Mourad Benamara, \\ and Min Zou ${ }^{1}$ \\ ${ }^{1}$ Department of Mechanical Engineering, University of Arkansas, Fayetteville, AR 72701, USA \\ ${ }^{2}$ Department of Physics and Astronomy, California State University Northridge, CA 91330, USA \\ ${ }^{3}$ Advanced Photon Source, Argonne National Laboratory, Lemont, IL 60439, USA \\ ${ }^{4}$ Institute for Nanoscience and Engineering, University of Arkansas, Fayetteville, AR 72701, USA
}

Correspondence should be addressed to Xiangbo Meng; xbmeng@uark.edu and Kah Chun Lau; kahchun.lau@csun.edu

Received 20 April 2021; Accepted 26 July 2021; Published 7 September 2021

Copyright (c) 2021 Xiangbo Meng et al. Exclusive Licensee Beijing Institute of Technology Press. Distributed under a Creative Commons Attribution License (CC BY 4.0).

\begin{abstract}
In this work, we for the first time developed a novel lithium-containing crosslinked polymeric material, a lithicone that enables excellent protection effects over lithium ( $\mathrm{Li}$ ) metal anodes. This new lithicone was synthesized via an accurately controllable molecular layer deposition (MLD) process, in which lithium tert-butoxide (LTB) and glycerol (GL) were used as precursors. The resultant LiGL lithicone was analyzed using a suite of characterizations. Furthermore, we found that the LiGL thichicone could serve as an exceptional polymeric protection film over Li metal anodes. Our experimental data revealed that the Li electrodes coated by this LiGL lithicone can achieve a superior cycling stability, accounting for an extremely long cyclability of $>13,600 \mathrm{Li}-$ stripping/plating cycles and having no failures so far in $\mathrm{Li} / \mathrm{Li}$ symmetric cells at a current density of $5 \mathrm{~mA} / \mathrm{cm}^{2}$ and an areal capacity of $1 \mathrm{mAh} / \mathrm{cm}^{2}$. We found that, with a sufficient protection by this LiGL coating, Li electrodes could realize long-term stable cyclability with little formation of Li dendrites and solid electrolyte interphase. This novel LiGL represents a facile and effective solution to the existing issues of $\mathrm{Li}$ anodes and potentially paves a technically feasible route for lithium metal batteries.
\end{abstract}

\section{Introduction}

Lithium (Li) metal is among the most attractive anodes of rechargeable batteries, ascribed to its extremely high capacity of $3860 \mathrm{mAh} / \mathrm{g}$ and the lowest negative electrochemical potential $(-3.04 \mathrm{~V}$ versus the standard hydrogen electrode) [1]. The first practice of $\mathrm{Li}$ anode has been witnessed 40 years ago [2]. Due to its dendritic growth and continuous formation of solid electrolyte interphase (SEI), however, Li metal has been prohibited from commercialization. Along with an ever-increasing interest in high-energy lithium metal batteries (LMBs) such as lithium-sulfur (Li-S) and lithiumoxygen $\left(\mathrm{Li}-\mathrm{O}_{2}\right)$ batteries in recent years, stable $\mathrm{Li}$ metal anodes now are undergoing intensive investigation. A variety of technical strategies have been reported to date, such as three-dimensional (3D) Li-hosting frameworks [3], electrolyte additives [4], solid-state electrolytes [5], and surface coatings $[6,7]$. Among these efforts, surface coating remains as an facile and effective route, and there have been different inorganic and polymeric coatings developed via wet chemistry and vapor-phase processes [6-11]. In this regard, molecular layer deposition (MLD) recently has emerged as a new research thrust, featuring its accurately controllable growth of polymeric films with unparalleled conformality and uniformity at moderate deposition temperatures of $\leq 300^{\circ} \mathrm{C}[6$, $12,13]$. The first practice of MLD on Li anodes was exposed in 2018 and so far, only a few polymeric MLD coatings have been reported, including polyurea [14], AlEG (EG: ethylene glycol) $[15,16]$, and ZrEG [17]. These polymeric films generally are electrically insulating and have better flexibility over inorganic films, contributing to better protection effects and thereby better performance of Li metal anodes. Stimulated by these compelling polymeric films via MLD, in this study, we attempted to develop three new processes of lithicones through coupling lithium tert-butoxide $\left(\mathrm{LTB}, \mathrm{LiO}^{t} \mathrm{Bu}\right.$ ) with one of three organic precursors, glycerol (GL), EG, and hydroquinone (HQ). Similar to other metalcones [12], lithicones are polymeric lithium alkoxides with carbon- 
containing backbones, i.e., -Li-O-R-O-Li-. Among these three resultant lithicones (LiGL, LiEG, and LiHQ), we particularly investigated the protective effects of LiGL on Li metal anodes as well as its MLD growth. Very encouragingly, the LiGL films, compared to the other MLD coatings [14-17] reported to date, enable a much longer cyclability in $\mathrm{Li} / \mathrm{Li}$ cells, accounting for over 13,600 Li-stripping/plating cycles $(>5,440 \mathrm{hrs})$ at an areal current density of $5 \mathrm{~mA} / \mathrm{cm}^{2}$ and around 5,500 Li-stripping/plating cycles $(\sim 5,500 \mathrm{hrs})$ at an areal current density of $2 \mathrm{~mA} / \mathrm{cm}^{2}$ both at an areal capacity of $1 \mathrm{mAh} / \mathrm{cm}^{2}$ without any signs to failure so far. Compared to previous surface-modified $\mathrm{Li}$ anodes as summarized in literature [8], to the best of our knowledge, these LiGL-coated $\mathrm{Li}$ electrodes in this work have exhibited the best performance to date. In Figure S1 in Supporting Information, the cyclability of our LiGL-coated Li electrodes is compared to those Li electrodes coated by other MLD films and by other coatings reported in literature. Our LiGL-coated Li electrodes showed a remarkable improvement in cyclability.

\section{Experimental}

2.1. MLD Processes. Using a commercial MLD system (Savannah 200, Ultratech Inc., MA) with argon (Ar) as the carrier gas, three MLD processes of lithicones have been investigated in this study, i.e., LiGL, LiEG, and LiHQ. LTB (Sigma-Aldrich, USA) was preheated in a bubbler at $150^{\circ} \mathrm{C}$ for a sufficient vapor supply, while EG (Sigma-Aldrich, USA), GL (Sigma-Aldrich, USA), and HQ (Sigma-Aldrich, USA) were maintained in stainless steel cylinders at 40 , 150 , and $150^{\circ} \mathrm{C}$, respectively. This MLD system was integrated with an Ar-filled glove box (having an oxygen and water concentration lower than $1 \mathrm{ppm}$ ), and this integration has greatly expanded the fabrication capability of the MLD system to many air-sensitive materials. In these three processes, LTB as the Li source was commonly used to couple with GL, EG (Sigma-Aldrich, USA), and HQ at $150^{\circ} \mathrm{C}$, respectively. The timing sequence of a single MLD cycle was typically in a sequence of $t_{1}-t_{2}-t_{3}-t_{4}$, corresponding to the LTB dose, the first Ar purge, the GL/EG/HQ dose, and the second Ar purge, respectively. The precursor dosing time $t_{1}$ was optimized as $3 \mathrm{~s}$, while $t_{3}$ was optimized as $0.15 \mathrm{~s}$ for EG, $2 \mathrm{~s}$ for GL, and $2 \mathrm{~s}$ for HQ. The purge time of $t_{2}$ and $t_{4}$ was optimized as $60 \mathrm{~s}$ for all these lithicones. During the MLD processes, the Ar gas flow was remained at $20 \mathrm{sccm}$.

These three MLD processes have been monitored using an in situ quartz crystal microbalance (QCM). The QCM studies were conducted using a gold sensor crystal (Inficon, USA). The crystal was sealed at the center of the MLD reactor lid. The doses of the different MLD precursors cause surface reactions with the growth of the targeted lithicones on the crystal surface. The growth of these three lithicones was monitored by the QCM to acquire time-resolved mass changes in $\mathrm{ng} / \mathrm{cm}^{2}$ and was recorded in digital data. To establish a uniform starting surface for these three MLD processes, we deposited an $\mathrm{Al}_{2} \mathrm{O}_{3}$ layer on the QCM surface via atomic layer deposition (ALD, an analogous technique of MLD exclusively for inorganics [12]) using trimethylaluminum
(TMA) and water as precursors with the timing sequence 0.05-10-0.025-10 s.

2.2. Material Characterization. We grew LiGL films over nitrogen-doped graphene nanosheets (N-GNS, ACS Material, USA), Si wafers (UniversityWafer, USA), steel plates, and Li chips (MTI, USA). The as-grown LiGL films were characterized using a suite of techniques. We observed the deposition of LiGL films over N-GNS using a scanning electron microscopy (SEM, XL30, Philips FEI) to determine the film thickness and thereby the growth per cycle (GPC). The SEM is equipped with an energy-dispersive $\mathrm{X}$-ray spectroscopy (EDX), which was used to detect the composition of the LiGL films. Synchrotron-based X-ray diffraction (XRD) was used to determine the crystallinity of the LiGL films and performed at the beamline 12-ID-D at Advanced Photon Source (APS) at Argonne National Laboratory (ANL, IL, USA). The X-ray wavelength was $0.5165833 \AA$. The scattering pattern was collected by two-dimensional photon counting area detector (Pilatus $100 \mathrm{~K}$ ). Two X-ray photoelectron spectroscopies (XPS) were used for analyzing sample compositions and element contents. LiGL films on Si wafers were analyzed using a Thermofisher Scientific K-Alpha XPS system having an $\mathrm{Al} \mathrm{K}_{\alpha}$ source $(1486.7 \mathrm{eV})$ at $12 \mathrm{kV}$ and $3 \mathrm{~mA}(36 \mathrm{~W})$ with a beam spot size of 400 micron. XPS depth profiling measurements were conducted using $\mathrm{Ar}^{+}$sputtering on Li and LiGL samples with a Versa Probe III XPS having a mono $\mathrm{Al} \mathrm{K}$ source $(1486.6 \mathrm{eV})$.

The surface topography, Young's modulus, and adhesion force of the LiGL coatings on steel plates were measured using the PeakForce quantitative nanomechanical (PFQNM) module of an atomic force microscope (AFM, Dimension Icon, Bruker USA). A RTESPA-300-30 AFM probe (Bruker, USA) with an end radius of $31 \mathrm{~nm}$ and a spring constant of $41.28 \mathrm{~N} / \mathrm{m}$ was used for the PFQNM mapping. An average of 3 different measurements, along with the property maps, is presented in this article.

2.3. Computational Simulations. Based on the XPS data of depth profiling on the LiGL film (Figure S4), the atomic distribution of $\mathrm{C}, \mathrm{H}, \mathrm{O}$, and $\mathrm{Li}$ is not homogeneous along the depth. Therefore, a range of $\mathrm{C}, \mathrm{H}, \mathrm{O}$, and $\mathrm{Li}$ stoichiometry is possible. To simulate the basic properties of this amorphous LiGL bulk, a predefined $\mathrm{C}, \mathrm{H}, \mathrm{O}$, and $\mathrm{Li}$ concentration in the simulation cell is needed as an approximation. Assuming $\left(\mathrm{CH}_{2} \mathrm{CHCH}_{2}\right)\left(\mathrm{O}_{3} \mathrm{Li}_{2}\right)$ as the basic stoichiometric unit, the initial configuration of the amorphous LiGL bulk was constructed through random insertion of lithium and depletion of hydrogen from the hydroxy group in randomly packed twelve glycerol $\left(\mathrm{C}_{3} \mathrm{H}_{8} \mathrm{O}_{3}\right.$ or $\left.\mathrm{CH}_{2} \mathrm{OH}-\mathrm{CHOH}-\mathrm{CH}_{2} \mathrm{OH}\right)$ molecules in a cubic simulation cell with length $11.50 \AA$. The $\left(\mathrm{CH}_{2} \mathrm{CHCH}_{2}\right)$ $\left(\mathrm{O}_{3} \mathrm{Li}_{2}\right)$ stoichiometry is likely on the top layer of LiGL films, according to the XPS data of depth profiling (Figure S4). In this work, all the electronic structure calculations were performed in the framework of density functional theory (DFT) based on the generalized gradient approximation (GGA) with Perdew-Burk-Ernzerhof (PBE) [18] functional under the projector augmented wave 
(PAW) method [19]. To account for the van der Waals interactions, Grimme's D2 method [20] was included in all calculations (i.e., PBE-D2). The plane wave energy cutoff was set to $400 \mathrm{eV}$, and the energy convergence criteria were set to $10^{-4} \mathrm{eV} /$ atom for all the ab initio molecular dynamic (AIMD) simulations. To accommodate a large simulation cell size (156 atoms), all the AIMD simulations have been conducted within a $(1 \times 1 \times 1) k$-mesh at $\Gamma$ point using the first-principle DFT program package VASP $[21,22]$. Additionally, to obtain thermodynamically stable configuration of the amorphous LiGL bulk structure at room temperature, the structures obtained from DFT optimizations were then thermally equilibrated at $T=300 \mathrm{~K}$, using AIMD simulations based on an Nose-Hoover NVTensemble with a total simulation time 50 ps and a time step of $1 \mathrm{fs}$.

2.4. Electrochemical Testing. The protective effects of the LiGL films were mainly investigated in $\mathrm{Li} / \mathrm{Li}$ symmetric cells using CR2032 coin cells. 500- $\mu \mathrm{m}$ thick Li chips were first punched into 7/16" disks and fixed on stainless steel spacers. Then, LiGL films of different thicknesses were deposited on these $\mathrm{Li}$ chips through controlling MLD cycles at $150^{\circ} \mathrm{C}$. The LiGL-coated Li chips were assembled into symmetric $\mathrm{Li} / \mathrm{Li}$ coin cells directly in the integrated MLD-glove box system. As controls, bare Li chips were also assembled into symmetric Li/Li cells in the glove box. The Celgard 2325 membrane was used as the separator, and the electrolyte was $1 \mathrm{M}$ bis(trifluoromethane)sulfonamide lithium salt (LiTFSI, Sigma Aldrich) dissolved in 1,3-dioxolane (DOL) and 1,2-dimethoxyethane (DME) (1:1, v/v) (Sigma Aldrich). The electrolyte has no other additives (e.g., $\mathrm{LiNO}_{3}$ ) in order to highlight the exceptional protection effects of the LiGL films. Each cell used $20 \mu \mathrm{L}$ of the electrolyte. Before electrochemical testing, all the symmetric cells were rested for 20 hours to stabilize their impedance [23]. At room temperature $\left(\sim 25^{\circ} \mathrm{C}\right)$, then, the symmetric cells were tested for Listripping/plating cyclability under different current densities $\left(2,5\right.$, and $\left.7.5 \mathrm{~mA} / \mathrm{cm}^{2}\right)$ while the stripping/plating capacity was fixed as $1 \mathrm{mAh} / \mathrm{cm}^{2}$. The battery cycler was a Neware BTS4000 Battery Testing System (Shenzhen, China). Measurements of electrochemical impedance spectroscopy (EIS) were conducted on $\mathrm{Li} / \mathrm{Li}$ symmetric cells in the range of $10 \mathrm{~m} \mathrm{~Hz}$ to $100 \mathrm{kHz}$ with an amplitude voltage of $5 \mathrm{mV}$ using a BioLogic SP-200 potentiostat.

\section{Results and Discussion}

3.1. The Growth of LiGL Films. In this study, we attempted three precursor couples for growing lithicones, i.e., LTBGL, LTB-EG, and LTB-HQ. Figure 1(a) illustrates the growth principle of these lithicones, exemplified by the MLD LiGL process using LTB and GL as precursors. One LiGL MLD cycle consists of four repeatable steps: LTB dose $/ 1^{\text {st }}$ purge/GL dose $/ 2^{\text {nd }}$ purge. We controlled the MLD cycles to achieve a desirable film thickness. We measured the growth of these MLD processes using an in situ QCM $[24,25]$. To establish a repeatable uniform starting surface, we predeposited an $\mathrm{Al}_{2} \mathrm{O}_{3}$ surface on the QCM crystals using ALD (inset of
Figure 1(b)), prior to performing the QCM measurements of the three MLD processes. The QCM measurements verified that all these precursor couples are feasible for growing lithicones, i.e., LiGL (Figures 1(b)-1(d)), LiEG (Figure S2a and $2 \mathrm{~b}$ ), and LiHQ (Figure $\mathrm{S} 2 \mathrm{c}$ and $2 \mathrm{~d}$ ). We were particularly interested in LiGL, for GL has three hydroxyl groups and is likely to form a crosslinked polymeric structure [26]. Our QCM measurements also revealed that the LiGL MLD process enables a much faster growth per cycle (GPC) (Figure 1(b)) than those of LiEG and LiHQ MLD processes. Consequently, we devoted to investigating the MLD process of LiGL and its effects on $\mathrm{Li}$ anodes. MLD QCM measurements in Figure 1(b) disclose that the LiGL growth on $\mathrm{Al}_{2} \mathrm{O}_{3}$ is nearly linear but can be divided into two regions: initiation and stable growth regions. Figures 1(c) and $1(\mathrm{~d})$ reveal more details in a single MLD cycle during the initiation and stable growth region, respectively, in which the doses of LTB and GL are signified by two different colored bars. Each dose of these two precursors caused some mass gain $\left(m_{1}\right.$ or $m_{2}$ as shown in Figure 1(d)) on QCM. The average mass gain $\left(\Delta m=m_{1}+m_{2}\right)$ is $\sim 200 \mathrm{ng} / \mathrm{cm}^{2} / \mathrm{cycle}$ in the initiation region $\left(\sim 30\right.$ cycles starting on an $\mathrm{Al}_{2} \mathrm{O}_{3}$ film, Figure S3) while $\sim 320 \mathrm{ng} / \mathrm{cm}^{2} /$ cycle in the stable growth region (Figure S3). The stable region exhibits a highly repeatable GPC. We deposited the LiGL films over one type of N-GNS (Figure 2(a)) to determine its GPC at $150^{\circ} \mathrm{C}$. The $\mathrm{N}-\mathrm{GNS}$ features its high surface area and thin wrinkles of $<3 \mathrm{~nm}$ [27]. Observing the thickness changes of the wrinkle of the N-GNS after 20 cycles (Figure 2(b)) of the LiGL deposition using an SEM, we could conclude that the average GPC of the MLD LiGL is $\sim 2.7 \mathrm{~nm} /$ cycle, which is among the highest GPCs of all the MLD processes reported to date. In addition to the SEM images, we conducted elemental mapping over the 20-cycle LiGL-coated NGS using an EDX (Figure 2(c)), which shows the distributions of $\mathrm{N}, \mathrm{C}$, and O. $\mathrm{N}$ is from N-GNS, C is from N-GNS and LiGL, and $\mathrm{O}$ is from LiGL. Consequently, EDX mapping revealed that the MLD LiGL coating over the N-GNS is very conformal and uniform.

According to our experience in MLD, we postulated the overall reaction of the MLD LiGL in Equation (1) as follows:

$$
\begin{aligned}
3 \mathrm{LiO}^{t} \mathrm{Bu} & +\left(\mathrm{CH}_{2} \mathrm{CHCH}_{2}\right)(\mathrm{OH})_{3} \longrightarrow\left(\mathrm{CH}_{2} \mathrm{CHCH}_{2}\right)(\mathrm{OLi})_{3} \\
& +3 \mathrm{HO}^{t} \mathrm{Bu} .
\end{aligned}
$$

Thus, the LiGL is supposed to have a unit structure of $\left(\mathrm{CH}_{2} \mathrm{CHCH}_{2}\right)(\mathrm{OLi})_{3}$ in its ideal MLD condition. The overall reactions of the MLD LiEG and LiHQ are described in Equation S1 and S2 in Supporting Information, respectively. To verify our postulation, we employed an XPS to determine the composition of the deposited LiGL films on Si wafers. In Figure 2(d), the $\mathrm{O} 1 \mathrm{~s}$ spectra show a strong peak at $529.6 \mathrm{eV}$ and a weak peak at $531.4 \mathrm{eV}$, assigned to $\mathrm{O}^{2-}$ in Li$\mathrm{O}$ bonds [28-30] and C-O-Li $[31,32]$, respectively. The C $1 \mathrm{~s}$ spectra show two evident peaks at 284.8 and $283.6 \mathrm{eV}$, which are related to $\mathrm{C}-\mathrm{C} / \mathrm{C}-\mathrm{H}[32-33]$ and $\mathrm{Li}-\mathrm{O}-\mathrm{C}$ groups $[32,34]$, respectively. The two weak peaks at 288.7 and 


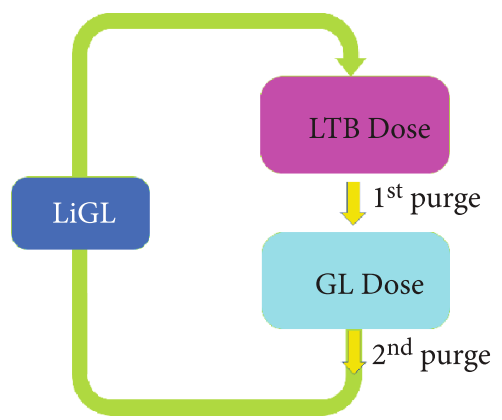

(a)

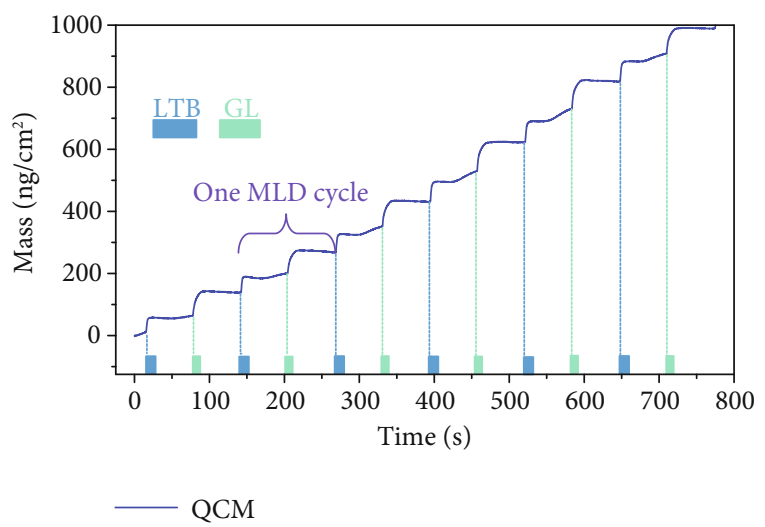

(c)

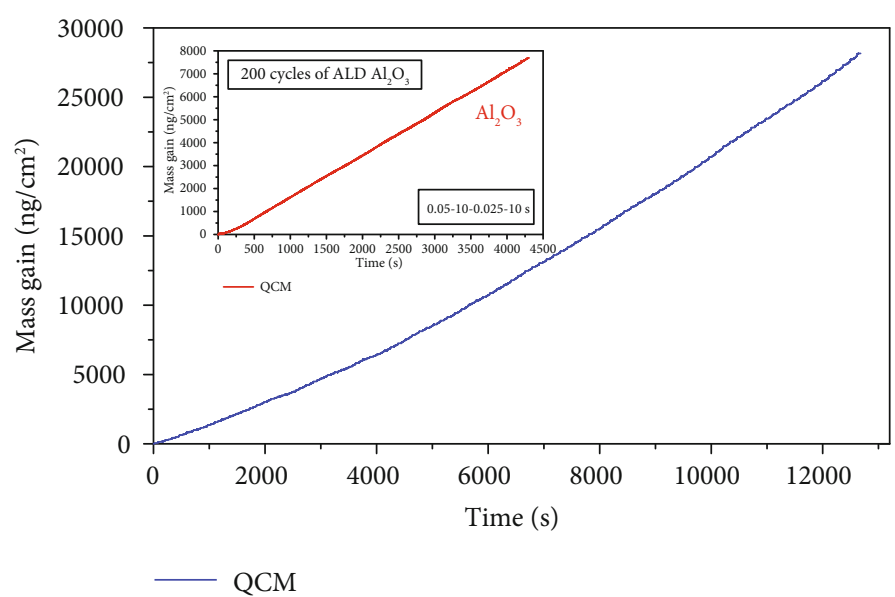

(b)

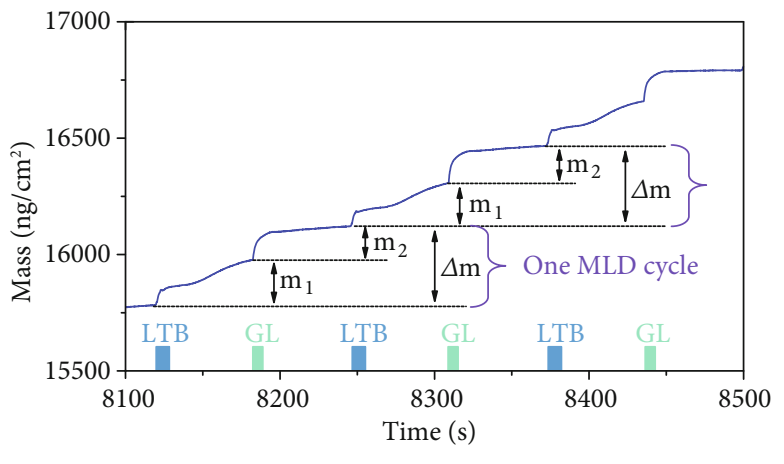

- QCM

(d)

FIGURE 1: Growth of MLD LiGL lithicone. (a) Illustration of the LiGL MLD process. (b) QCM measurements of MLD LiGL in 100 cycles at $150^{\circ} \mathrm{C}$ (inset: the QCM growth of 200-cycle ALD $\mathrm{Al}_{2} \mathrm{O}_{3}$ prior to the MLD LiGL growth). (c) The QCM profile of the MLD LiGL in the first 10 cycle during the initiation region. (d) The QCM profile of the MLD LiGL in the stable growth region.

$287.1 \mathrm{eV}$ are related to $\mathrm{O}=\mathrm{C}-\mathrm{OH}$ [35] and $\mathrm{C}-\mathrm{O}$ bonds [30], respectively. The Li $1 \mathrm{~s}$ XPS spectra show only one peak at $53.3 \mathrm{eV}$, and this should be attributed to Li-O $[29,36]$. According to the XPS analyses, the deposited LiGL contains 31.69 at. $\%$ of $\mathrm{Li}, 28.73$ at. $\%$ of $\mathrm{C}$, and 39.59 at.\% of O (more details are in Table S1). The element contents of $\mathrm{Li}, \mathrm{C}$, and $\mathrm{O}$ are basically consistent to our postulation on the LiGL unit structure, $\left(\mathrm{CH}_{2} \mathrm{CHCH}_{2}\right)(\mathrm{OLi})_{3}$. However, we also noticed that there are less $\mathrm{C}$ and more $\mathrm{O}$ than those of our postulation. This might be due to some side reactions and incomplete surface reactions. Such cases have been widely reported in previous ALD and MLD studies [12, 37]. To further gain some insight on the evolution of the LiGL films with film thickness, we utilized XPS to conduct depth profiling over a 75-MLD-cycle LiGL film on an Si wafer (Figure S4). It was revealed that the composition of the LiGL film varies with film thickness. For example, the Li content changes from $\sim 20$ at.\% at the top film surface to $\sim 50$ at.\% at the interface between the LiGL film and the $\mathrm{Si}$ wafer. Two reasons might have contributed to this phenomenon: (i) the influence of substrates (e.g., Si wafers) and (ii) the influence of the successor MLD cycles on the predecessor MLD cycles. The former has been widely noticed while the latter was also reported previously [38]. We will invest some special effort on these topics in our future research. Additionally, we did some preliminary study on the air stability of the as-deposited LiGL and measured the XPS spectra of an LiGL film exposed in air for $1 \mathrm{~h}$ (more details are in Table S2). The effects of $1 \mathrm{~h}$ air exposure were compared in Figure S5 and discussed in Supporting Information. The information revealed that the as-deposited LiGL is sensitive to air to some extent, such as water. In addition, synchrotron-based XRD has been conducted on the LiGL films grown on N-GNS and revealed no pronounced crystallinity with the LiGL films (very broad diffuse scattering peak shown in Figure S6). Thus, the LiGL films are amorphous. Moreover, we computed the amorphous LiGL bulk structures using AIMD simulation, and the calculated radial distribution function (RDF) confirmed the dominance of Li-O, C-O, C$\mathrm{H}$, and $\mathrm{C}-\mathrm{C}$ bonds in the LiGL films (Figure S7).

3.2. The Superior Performance of the LiGL-Coated $L i / L i$ Symmetric Cells. To investigate the protective effects of the MLD LiGL films on Li metal anodes, we conducted a series of comparative studies on bare (uncoated) and LiGL-coated 


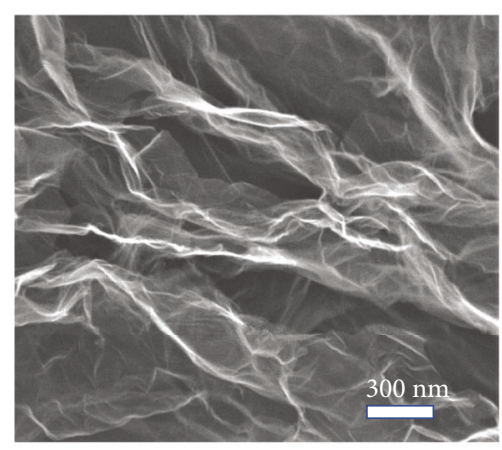

(a)

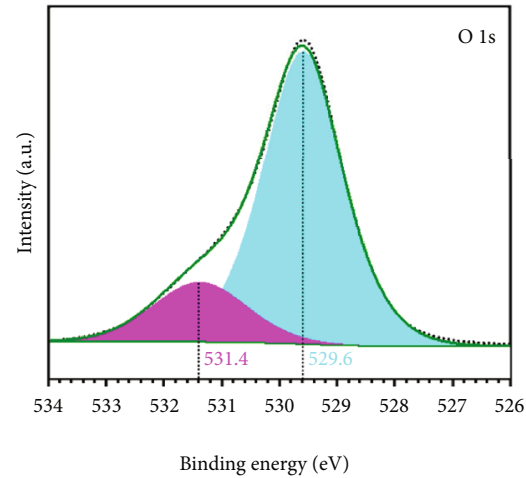

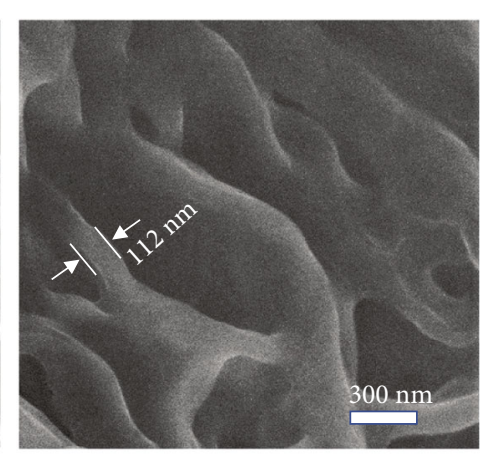

(b)

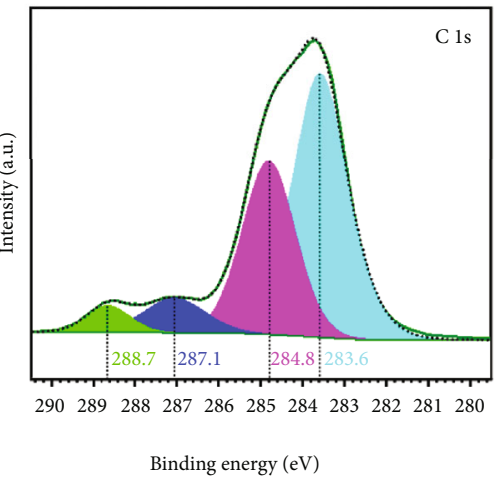

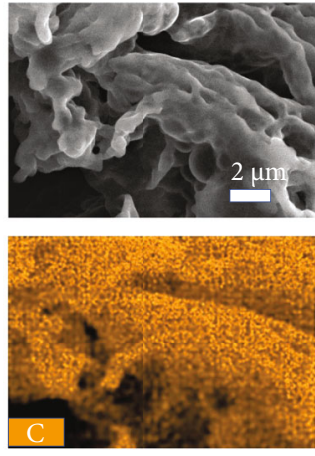

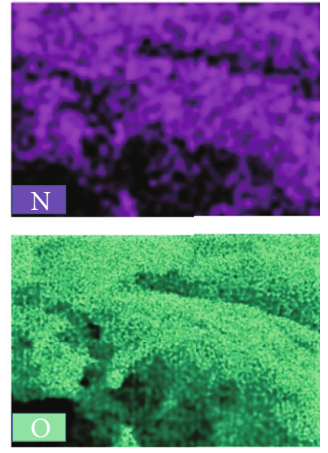

(c)

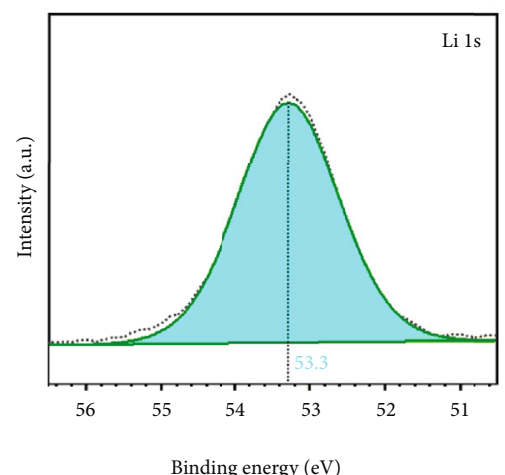

(d)

Figure 2: (a) SEM image of pristine N-GNS. (b) 20-MLD-cycle LiGL over N-GNS. (c) EDX mapping on 20-MLD-cycle LiGL supported by N-GNS. (d) High-resolution XPS analyses of LiGL films: O 1 s, C 1 s, and Li 1 s spectra.

Li metal electrodes in Li/Li symmetric cells. With a fixed areal capacity of $1 \mathrm{mAh} / \mathrm{cm}^{2}$, these $\mathrm{Li} / \mathrm{Li}$ cells were tested and compared for Li-stripping/plating cyclability under different current densities, i.e., 2,5 , and $7.5 \mathrm{~mA} / \mathrm{cm}^{2}$. These tests showed that the LiGL coatings were extremely effective and exhibited exceptional protective effects on Li metal electrodes (as illustrated in Figure 3). The Li metal electrodes were coated with LiGL of different MLD cycles and thereby named as LiGL- $X$ ( $X=$ MLD cycles $)$ for simplification. Specially, LiGL-0 indicates the bare Li electrode. Under $2 \mathrm{~mA} / \mathrm{cm}^{2}$ (Figure 3(a)), our tests revealed that a LiGL coating of $>10$ MLD cycles is necessary to achieve a desirable protection. The LiGL0/LiGL-0 cell (i.e., the cell with two bare Li electrodes symmetrically) could only sustain for less than 600 cycles. In comparison, the LiGL-10/LiGL-10 cell improved the cyclability to over 1,000 cycles but did not survive longer. In contrast, the LiGL-15/LiGL-15 cell could realize an exceptional cyclability up to 4,800 Li-stripping/plating cycles without any failure (Figure 3(a)). The overpotential of the LiGL-15/LiGL15 cell evolved slowly with Li-stripping/plating cycles. In the first 1400 cycles, the cell overpotential has been gradually increasing from 50 to $250 \mathrm{mV}$. Thereafter, the cell overpotential was relatively stable in the range of 250$300 \mathrm{mV}$. When the LiGL coating thickness was increased to 20 MLD cycles, more impressively, the LiGL-20/LiGL-20 cell (Figure 3(a) and Figure S8) also have achieved over 4,800 Listripping/plating cycles without any failure but had a much lower overpotential, which evolved from $\sim 100$ to $125 \mathrm{mV}$ in the first 500 cycles and then remained in the range of $\sim 125$ $140 \mathrm{mV}$. Even more intriguingly, we further investigated the performance of LiGL-60 and LiGL-90. The LiGL-60/LiGL60 and LiGL90/LiGL-90 cells have accomplished a cyclability of $\sim 5,500$ cycles without any failure (Figure 3(a) and Figure S8). Particularly, thicker coatings contributed to lower overpotentials. The overpotential of the LiGL60/LiGL-60 cell evolved from $\sim 75 \mathrm{mV}$ in the first 200 cycles and then gradually increased and stabilized at $\sim 140 \mathrm{mV}$ in the subsequent cycles. In comparison, the LiGL90/LiGL-90 cell started with an overpotential of $\sim 55 \mathrm{mV}$ in the first 100 cycle, gradually dropped the overpotential to $\sim 35 \mathrm{mV}$ in the subsequent 100 cycles, and then slowly increased to $\sim 60 \mathrm{mV}$ in the following cycling of over 5,000 cycles. All these intriguing results clearly demonstrated that the MLD LiGL coatings are exceptional in helping achieve superior Li metal anodes. Insets of Figure 3(a) and Figure S8 have shown the stripping/plating profiles of some cycles during different cycling periods.

To further verify the protective effects of the MLD LiGL coatings, we applied a much higher current density of $5 \mathrm{~mA} / \mathrm{cm}^{2}$ (Figure 3(b)). Our tests revealed that a bare $\mathrm{Li} / \mathrm{Li}$ cell (i.e., LiGL-0) sustained a low overpotential of $<130 \mathrm{mV}$ in the first $\sim 350$ cycles, but then exhibited a constant increase of cell overpotential and failed after $\sim 1,300 \mathrm{Li}$-stripping/plating cycles. In comparison, the LiGL-15/LiGL-15 cell has remained a low overpotential of $<130 \mathrm{mV}$ in the first 500 cycles, but then had a continuously evident increase in cell 

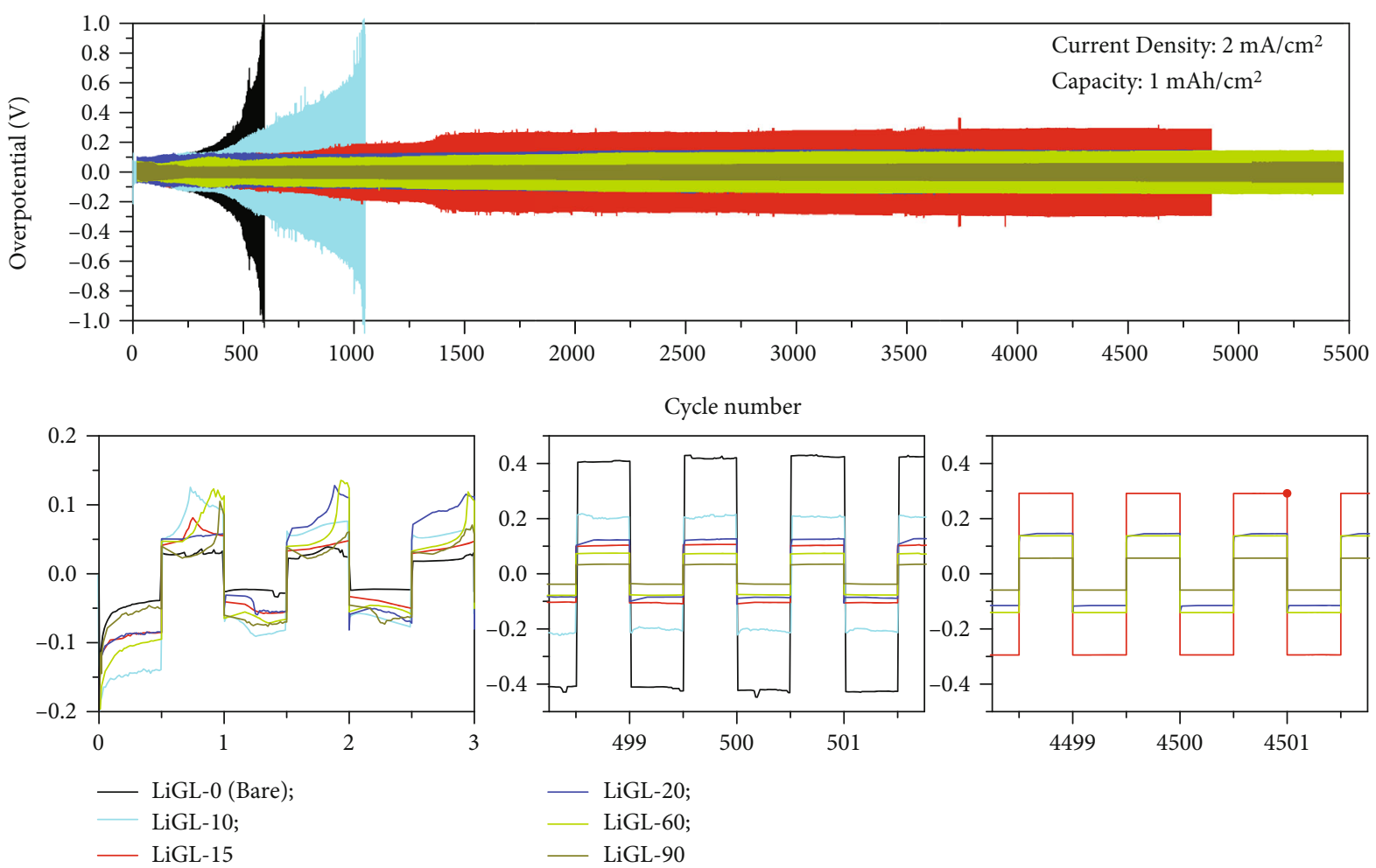

(a)
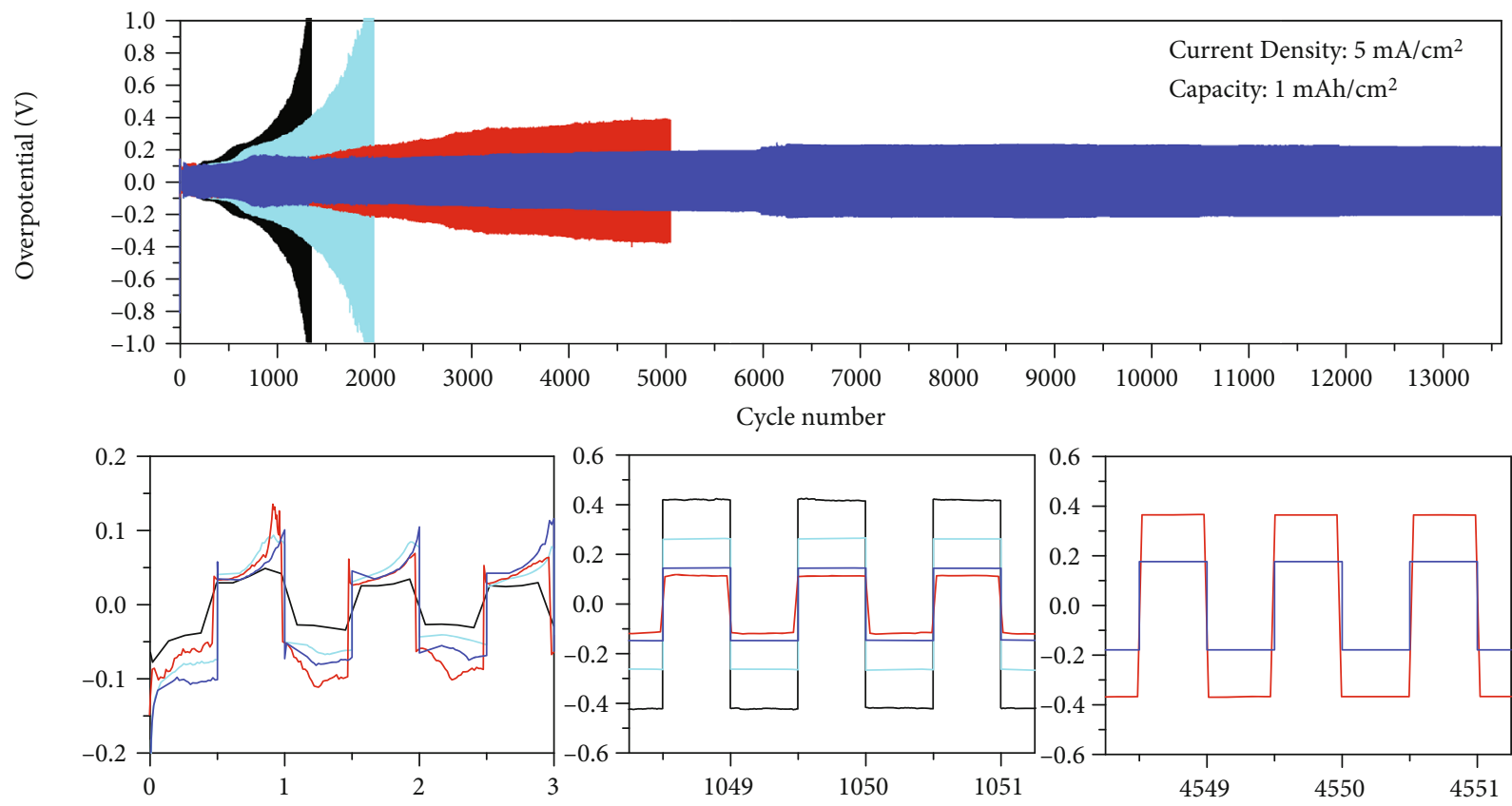

- LiGL-0 (Bare);

LiGL-15

— LiGL-20;

— LiGL-60

(b)

Figure 3: Electrochemical evaluations of LiGL-coated Li electrodes in $\mathrm{Li} / \mathrm{Li}$ symmetric cells. $\mathrm{Li} / \mathrm{Li}$ cells tested at (a) $2 \mathrm{~mA} / \mathrm{cm}^{2}$ and $(\mathrm{b})$ $5 \mathrm{~mA} / \mathrm{cm}^{2}$ with a fixed areal capacity of $1 \mathrm{mAh} / \mathrm{cm}^{2}$. 

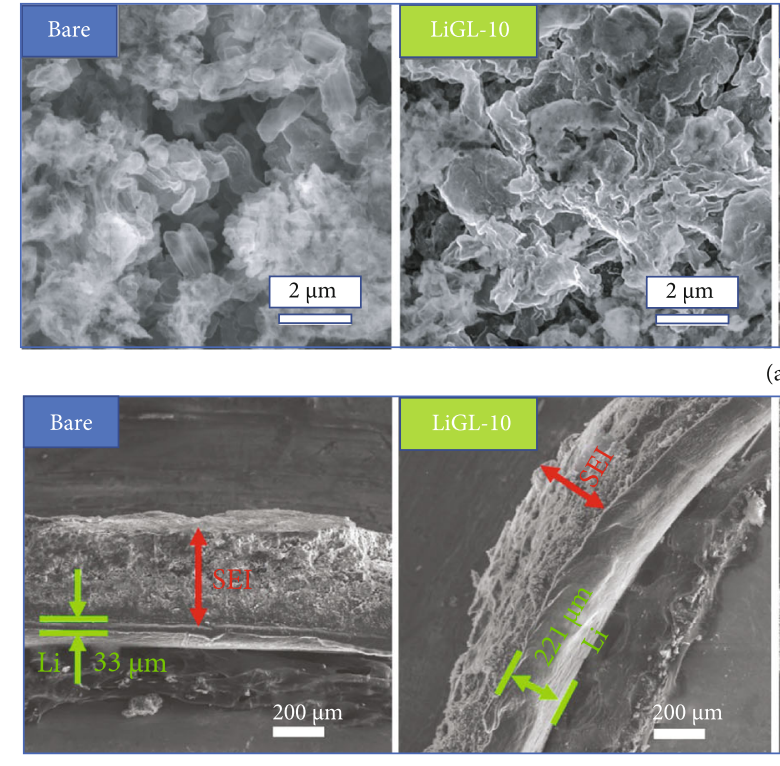
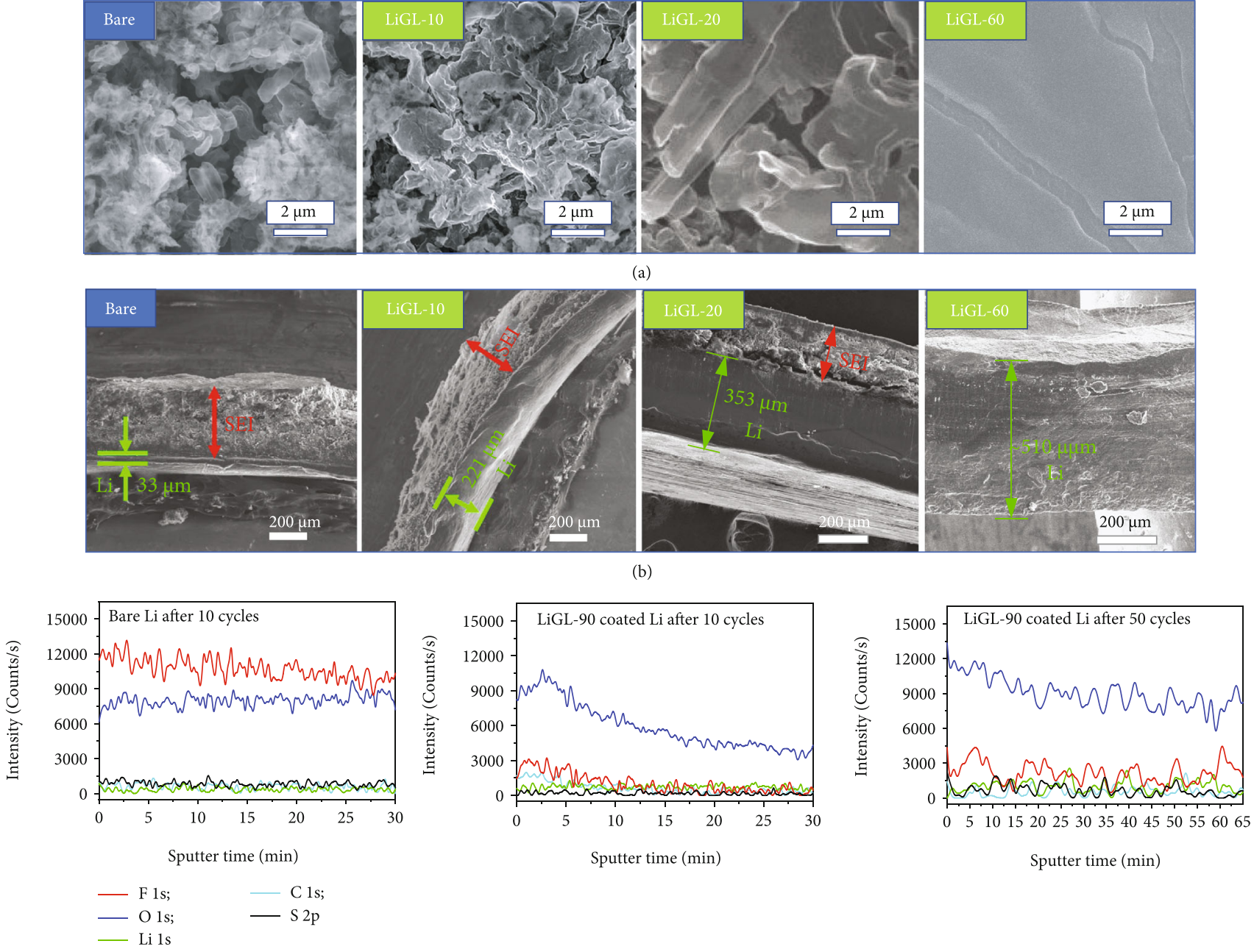

(c)

(d)

(e)

FIGURE 4: Analyses on cycled Li/Li cells. SEM images of (a) the surfaces and (b) the cross-sections of the cycled bare, LiGL-10, LiGL-20, and LiGL-60 electrodes after 700 Li-stripping/plating cycles. XPS depth profiling on (c) bare and (d, e) LiGL-90 electrodes after (c, d) 10 and (e) 50 Li-stripping/plating cycles.

overpotential and failed after $\sim 2,000$ cycles. The results indicated that both the LiGL-0/LiGL-0 and LiGL15/LiGL-15 cells probably suffered from uncontrollable formation of SEI or/and Li dendritic growth. In contrast, the LiGL-20/LiGL20 cell sustained a stable overpotential of $<130 \mathrm{mV}$ in $\sim 1,000$ cycles and then exhibited a gradually increasing overpotential from $\sim 130 \mathrm{mV}$ at $\sim 1,000^{\text {th }}$ cycle to $\sim 370 \mathrm{mV}$ after 5,000 cycles. More details have been shown in Figure S9. Very impressively, the LiGL-60/LiGL-60 cell has accomplished over 13,600 Li-stripping/plating cycles with a much smaller overpotential from $\sim 130 \mathrm{mV}$ at $\leq 1,000$ cycles to $\sim 220 \mathrm{mV}$ after 13,600 cycles. Very encouragingly, the LiGL-60/LiGL60 cell has not shown any failure and is still under testing. The excellent performance of the LiGLcoated $\mathrm{Li} / \mathrm{Li}$ cells was compared with the results reported in literature [8]. To the best of our knowledge, our LiGL/LiGL cells have set a record on the best cyclability reported so far. We even further investigated the effects of LiGL coatings at a much higher current density to $7.5 \mathrm{~mA} / \mathrm{cm}^{2}$ at an areal capacity of $1 \mathrm{mAh} / \mathrm{cm}^{2}$. Our results (Figure S10) show that the LiGL-90/LiGL-90 cell performed the best. These results seem to suggest that a thicker coating is more favorable for achieving stable cyclability at a higher current density. As for the underlying mechanism for the better effects of thicker LiGL coatings, we will discuss in the following parts in this study.

3.3. Understanding the Protective Effects of LiGL Coatings. To understand the protective effects of the LiGL coatings, we investigated some cycled cells: LiGL-coated and uncoated (bare). In Figure 4(a), we observed the morphologies of four Li electrodes (bare (i.e., LiGL-0), LiGL-10, LiGL-20, and LiGL-60) from cells after 700 Li-stripping/plating cycles at $2 \mathrm{~mA} / \mathrm{cm}^{2}$ and $1 \mathrm{mAh} / \mathrm{cm}^{2}$. Apparently, there were dendrite-like structures formed on the bare Li electrode. The dendrite-like structures were also observed on the LiGL-10 and LiGL-20 electrodes. In sharp contrast, the surface of the LiGL-60 electrode was featureless and smooth but has some fractures. The smooth surface was deemed to be the LiGL coating while the fractures were due to the 
broken LiGL film. This indicates that the 60-MLD-cycle LiGL film is sufficient to inhibit Li dendritic growth and protect the ether electrolyte from decomposition. More information is shown in Figure S11 about the surface of the LiGL-60 after $700 \mathrm{Li}$ stripping/platting. We observed that the LiGL-60 coating has broken into small pieces of $10-50 \mu \mathrm{m}$ but the protected Li electrode surface is clean. In other words, there has no $\mathrm{Li}$ corrosion observed on the LiGL-60 electrode. This also has been further verified by our observations on the cross-sections of the bare (LiGL-0), LiGL-10, LiGL-20, and LiGL-60 electrodes using SEM after 700 Listripping/plating cycles (Figure 4(b)). Results showed that, compared to the Li metal before cycling (Figure S12), the bare electrode has significantly corroded with the remarkable formation of SEI. In comparison, the LiGL-10 and LiGL-20 electrodes both have been evidently mitigated for their corrosions and have less formation of SEI. Thus, we concluded that the thicker the LiGL coatings, the better the protection effects. Very excitingly, the cross-section of the LiGL-60 is nearly intact without evident SEI layers and Li dendrites after cycling (Figure 4(b)).

Furthermore, we employed XPS depth profiling to investigate the composition evolution with film depth on three $\mathrm{Li}$ electrodes, one bare $\mathrm{Li}$ after $10 \mathrm{Li}$-stripping/plating cycles (Figure 4(c)), one LiGL-90 electrode after $10 \mathrm{Li}$-stripping/plating cycles (Figure 4(d)), and one LiGL-90 electrode after $50 \mathrm{Li}$-stripping/plating cycles (Figure 4(e)). In Figure 4(c), the XPS depth profiling data of the bare Li electrode reveal that, after $10 \mathrm{Li}$-stripping/plating cycles, the $\mathrm{F}$ and $\mathrm{O}$ signals are considerable and relatively stable with film depth in the 30 min sputter time. All the other signals of $\mathrm{Li}, \mathrm{C}$, and $\mathrm{S}$ are much weaker. These results confirm that there has a very thick SEI layer formed on the bare $\mathrm{Li}$ after $10 \mathrm{Li}$-stripping/plating cycles. The $\mathrm{F}$ and $\mathrm{O}$ elements are most probably from the decomposed LiTFSI salt and the ether solvent. More details are shown by the high-resolution XPS spectra of each element of the cycled bare electrode (Figure S13a). In sharp contrast, the $\mathrm{F}$ signal is detectable in the first 15 min sputter with a significantly reduced intensity on the LiGL-90 electrode after $10 \mathrm{Li}$-stripping/plating cycles (Figure 4(d)). Along with the evolution of the F signal, on the other hand, the $\mathrm{O}$ and $\mathrm{C}$ signals show similar evolutions, which drop and then level off. These results indicate that there has much less SEI formed on the LiGL-90 electrode. Some similar data are verified on the LiGL-90 electrode after 50 cycles (Figure 4(e)). This again corroborates that the LiGL films are chemically inert and can be used as an exceptional protective film in the ether electrolyte. More details are shown by the high-resolution XPS spectra of the cycled LiGL-90 electrode (Figure S13b).

Intrigued by the results of the LiGL-60 electrode (Figure 4 and S11), we conducted an interesting study to further investigate the protective effects of the LiGL coatings. In the study, a bare Li/Li cell and an LiGL-60/LiGL60 cell both were performed one $24 \mathrm{~h}$ stripping on one electrode at a current density of $2 \mathrm{~mA} / \mathrm{cm}^{2}$ (i.e., an areal capacity of $48 \mathrm{mAh} / \mathrm{cm}^{2}$ ), which corresponded to one $24 \mathrm{~h}$ plating on the opposite electrode. Then, the two bare Li electrodes and the two LiGL-60 electrodes were observed using SEM (Figure 5). The bare Li electrode after the $24 \mathrm{~h}$ stripping (Figures 5(a)(i)) was covered by craters (as circled by dashed yellow lines) and bumps (the areas other than the yellow circles). The craters are smooth while the bumps are decorated with numerous microwells (or microholes) mainly in the range of $10-50 \mu \mathrm{m}$. These microwells are further enlarged to show more details (Figure 5(a)(ii)-(iv)). One can clearly see from Figure 5(a)(iv) that these microwells contain many dendritic structures (or micropillars). We postulated that the craters were the areas that have stripped Li first and then ceased while the bumps were the areas that did not strip $\mathrm{Li}$ at the very beginning but became the new areas for stripping after the craters ceased stripping. Thus, the stripping process on bare $\mathrm{Li}$ electrodes is not uniform, and the stripping areas change with time. On the other hand, the opposite bare $\mathrm{Li}$ after a $24 \mathrm{~h}$ plating was also observed (Figure 5(b)). Very strikingly, there is a large amount of dendritic Li deposited on the bare Li surface (Figure 5(b)(i)). It could be seen from Figure 5(b)(ii) that the deposited Li was separated from the originally bare Li while the originally bare Li surface was intact (Figure 5(b)(iii)). The deposited Li (Figure 5(b)(iv)) was in micron-sized dendritic structures that were squeezed together with clear boundaries. We believed that the bare Li surface and the dendritic structures have been covered by one layer of SEI, and the SEI layers have separated them from each other. The formation of this SEI layer consumed $\mathrm{Li}$ and the electrolyte.

In sharp contrast, the LiGL-60 electrode after $24 \mathrm{~h}$ stripping (Figure 5(c)) was free of craters and bumps but was covered by a uniform LiGL coating with numerous fractures. The fracturing gaps between the broken pieces of the LiGL coating are $\sim 1 \mu \mathrm{m}$. Similarly, the opposite LiGL-60 electrode after $24 \mathrm{~h}$ plating (Figure 5(d)) was generally clean and smooth, but some areas have lost the LiGL coating with holes. These holes indicate that $\mathrm{Li}$ has deposited underneath the LiGL coating. Based on these observations in Figure 5, we concluded that the LiGL coating is ionically conductive and electronically insulating. This has been further verified and will be discussed in a later part in this study.

We further examined the protective effects of the LiGL coating after one $48 \mathrm{~h}$ stripping-plating cycle (i.e., 24 hours for stripping and then 24 hours plating). The bare Li after $48 \mathrm{~h}$ stripping-plating (Figure 6(a)) exhibited the similar morphology as shown in Figure 5(b), covered with a thick dendritic Li layer on the top of the bare Li electrode. The opposite bare $\mathrm{Li}$ after $48 \mathrm{~h}$ plating-stripping (Figure 6(b)) has a similar appearance as shown in Figure 5(a), decorated with numerous craters and bumps covered by many microwells. During the stripping/plating processes, unavoidably, the bare Li electrodes were exposed to the electrolyte with uncontrollable formation of SEI and consumption of the electrolyte and Li. Very differently, the LiGL-60 electrode after $48 \mathrm{~h}$ stripping-plating (Figure 6(c)) was clean on its surface and covered with numerous small pieces of LiGL coatings mainly in the range of $10-50 \mu \mathrm{m}$. The fractures between the LiGL pieces are 1-2 $\mu \mathrm{m}$. Similarly, the LiGL-60 electrode after $48 \mathrm{~h}$ plating-stripping (Figure 6(d)) was clean and covered with small pieces of the LiGL film. It needs to particularly point out that, after one $48 \mathrm{~h}$ cycle of 


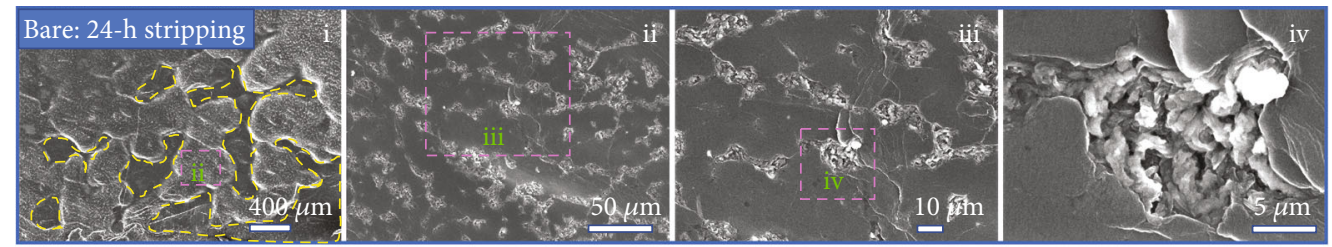

(a)

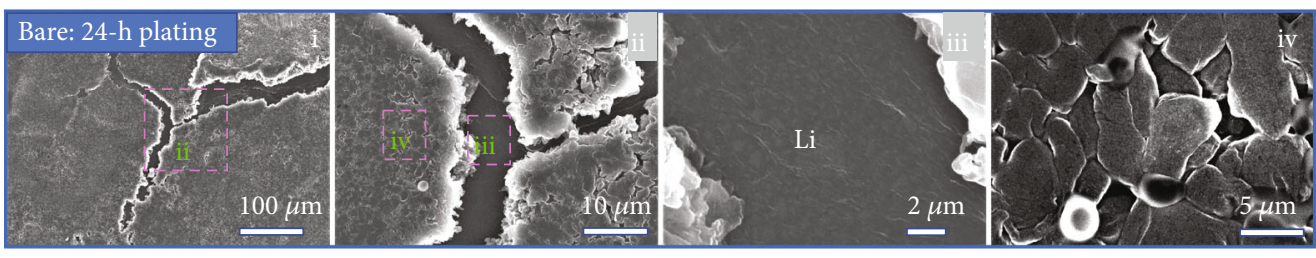

(b)

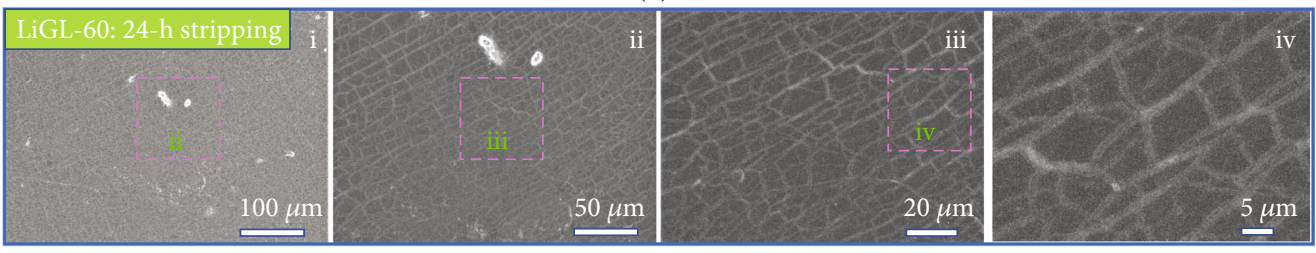

(c)

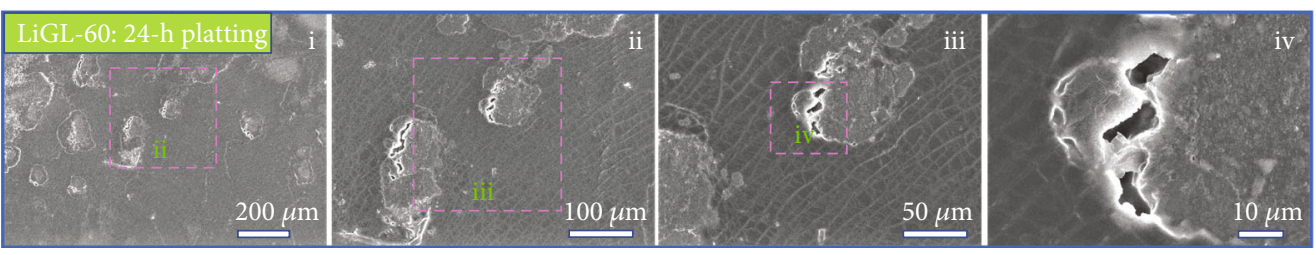

(d)

Figure 5: SEM observations of the morphological changes of Li electrodes. (a, b) The bare Li electrode and (c, d) the LiGL-60 electrode after one $24 \mathrm{~h}$ stripping (or plating) at $2 \mathrm{~mA} / \mathrm{cm}^{2}$.

stripping/platting, the bare $\mathrm{Li} / \mathrm{Li}$ cell was completely dried after the cell was opened, and the separator was tightly pinned on one electrode, while the LiGL-60/LiGL-60 cell was wet with sufficient electrolyte when it was opened. Thus, we are confident that the LiGL coatings with suitable thicknesses are very effective to protect Li electrodes from dendritic growth and SEI formation.

To further clarify the effect of the LiGL coating thickness, we also observed the bare $\mathrm{Li} / \mathrm{Li}$ and LiGL-20/LiGL-20 cells after one stripping (or plating) at a current density $2 \mathrm{~mA} / \mathrm{cm}^{2}$ and a capacity of $1 \mathrm{mAh} / \mathrm{cm}^{2}$ (Figure S14). No surprise, the bare $\mathrm{Li}$ was covered by dendritic Li on the plating side (Figure S14a) while the bare Li was decorated with craters and bumps on the stripping side (Figure S14b). In contrast, the LiGL-20 electrodes were clean. Specifically, there were no dendritic structures on the plating side (Figure S14c), and there had no crates and bumps on the stripping side (Figure S14d). Compared to the LiGL-60 coating, however, the LiGL-20 coating was prone to break into smaller pieces compared to the LiGL-60 coating (Figures 5 and 6). Thus, thicker LiGL coatings are beneficial to protect $\mathrm{Li}$ metal electrodes and thereby realize better cyclability, as shown in Figures 3 and 4.

To achieve high energy LMBs, particularly, thin Li films (50 $\mu \mathrm{m}$ or thinner) are required $[39,40]$. In this regard, we have confirmed that our LiGL coatings could be scaled up at ease and have been deposited on large-scale thin Li films
( $\sim 5 \mu \mathrm{m}$ ) uniformly for making pouch cells. The results from pouch cells will be reported somewhere else.

3.4. Understanding the Properties of LiGL Coatings. Evidently, the LiGL coatings have exceptional protection effects on Li metal electrodes. Therefore, their properties are of significance, such as mechanical properties and conductivities. In this regard, we employed AFM to determine the topography and nanomechanical properties of the LiGL-100 and -200 coatings using PFQNM (Figure 7). The LiGL-100 and -200 coatings were deposited on the cell spacers (stainless steel plates). The LiGL-100 coating exhibited a porous structure over the mapped area (Figure $7(\mathrm{a})(\mathrm{i})$ ), and the average roughness of the coating is $31.4 \pm 4.1 \mathrm{~nm}$. Although Young's modulus map showed a uniform stiffness throughout the coated area, the porous region of the coating exhibited a higher Young's modulus due to the less defined contact area and the probable interaction between the AFM tips and the steel substrate (Figure 7(a)(ii)). The average Young's modulus of the coating in the whole mapped area is $5.82 \pm 0.89$ $\mathrm{GPa}$. Similar to Young's modulus map, the adhesion maps showed general uniformity but higher adhesion in the porous area of the coating. The average adhesion of the LiGL coating is $145.1 \pm 21.40 \mathrm{nN}$ (Figure 7(a)(iii)). The boxed area with the white dashed lines in Figure 7(a)(ii) and (iii) shows no porosity, and there is a significant difference in the average 


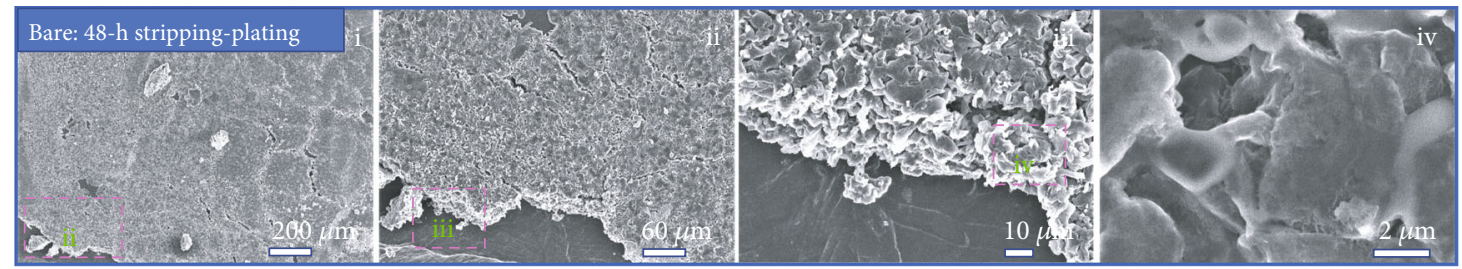

(a)

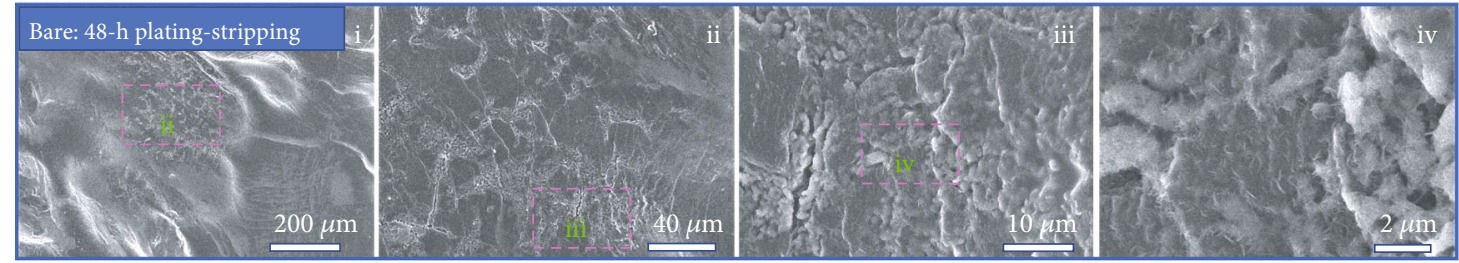

(b)

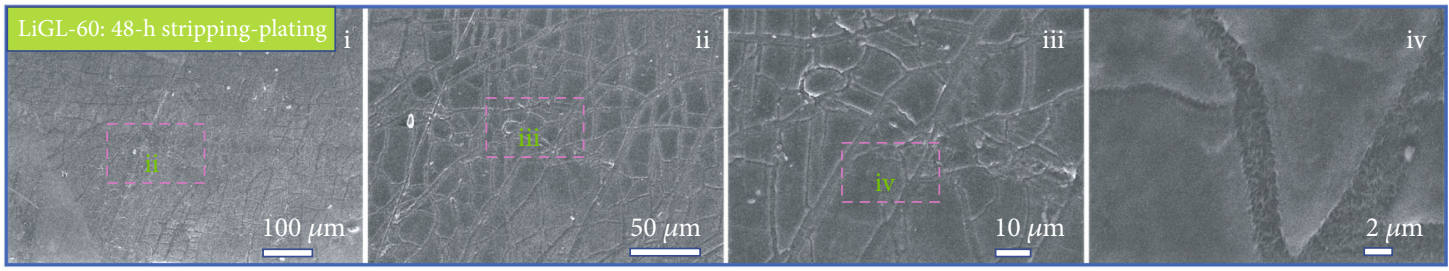

(c)

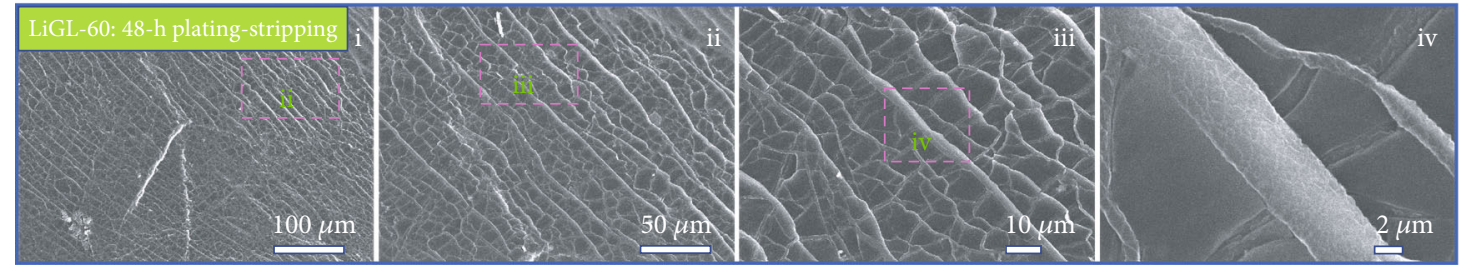

(d)

Figure 6: SEM observations of the morphological changes of Li electrodes. (a, b) The bare Li electrode and (c, d) the LiGL-60 electrode after one $48 \mathrm{~h}$ stripping-plating or platting-stripping at $2 \mathrm{~mA} / \mathrm{cm}^{2}$.

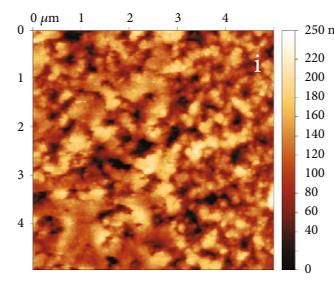

$\begin{array}{lllll}0 \mu \mathrm{m} & 1 & 2 & 3 & 4\end{array}$

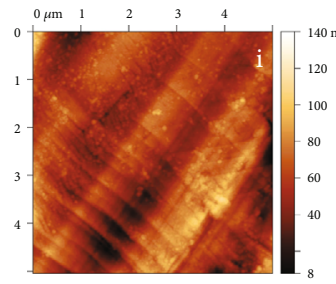

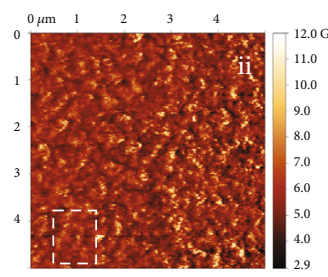

(a)

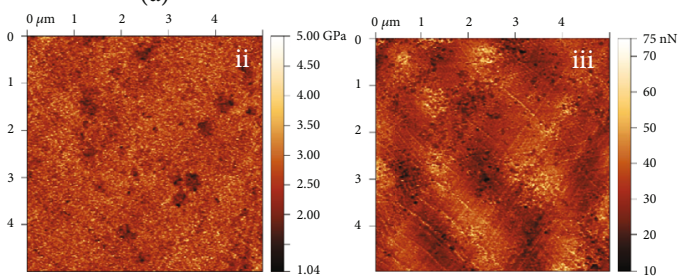

(b)

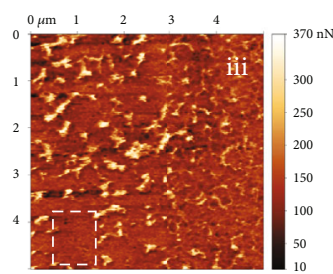

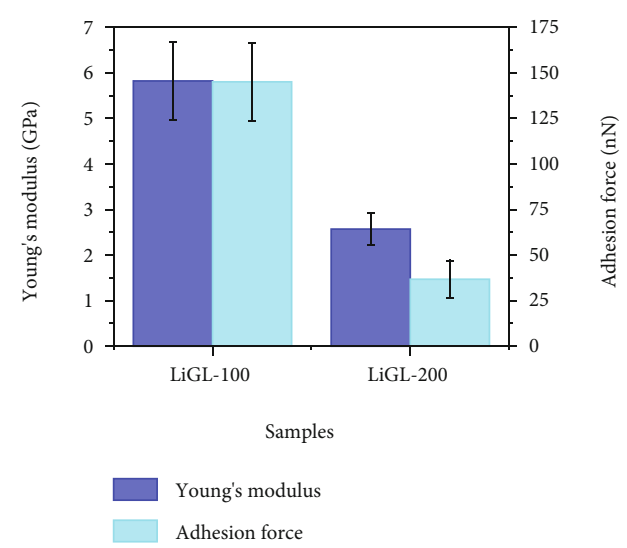

(c)

FIgURE 7: AFM measurements of the surface topography, Young's modulus, and adhesion force. (a) 100-cycle and (b) 200-cycle LiGL coating over steel plates. (c) Comparison of Young's modulus and adhesion force of these two samples. 
Young's modulus (3.24 GPa) and adhesion force $(77 \mathrm{nN})$ between this and the porous areas of the coating. We also investigated the LiGL-200 coating which showed much more uniform topography than the LiGL-100 coating (Figure 7(b)(i)). Its nanomechanical properties are also consistent throughout the mapped area. Figure 7(c) shows the comparison of nanomechanical properties of the LiGL100 and LiGL-200 coatings. The LiGL-100 coating shows higher average Young's modulus and adhesion than those of the LiGL-200 coating, due to the porosity and substrate effect on the thinner coating.

Compared to the Young's modulus of Li metal $(7.82 \mathrm{GPa})$ [41], these results revealed that the LiGL coatings are moderate in their mechanical properties. This underlies why the LiGL coatings are vulnerable to mechanical forces and easy to fracture. We conducted a comparative study through assembling a bare Li/Li cell and a LiGL-20/LiGL-20 cell and then opening them to observe their surface changes. We found that the bare Li after the press in assembling changed from a flat surface (Figure S15a) to a waved surface (Figure S15b and S15c) locally covered with numerous scaled patterns (Figure S15d-S15f). These patterns are several microns in size. On the other hand, we observed that the LiGL-20 electrode after the press in assembling has broken from a smooth surface (Figure S16a) into scaled patterns of several microns (Figure S16b-S16d). All these results clearly evidenced that the LiGL coatings were broken mainly during mechanical assembling but fairly stable during Li-stripping/stripping cycles. These also explained that thicker LiGL coatings could better protect Li.

In addition to the mechanical properties of the LiGL coatings, their electrical and ionic conductivity are of particularly significance. In this regard, we conducted experimental measurements and computational simulations. From DFT calculation, the amorphous LiGL is found to be electronically insulating with $\sim 3.0 \mathrm{eV}$ in band gap, as shown in the electronic density of states close to the Fermi level (Figure 8(a)). To explore the stability of electronic properties of the amorphous LiGL bulk at room temperature, we investigated the evolution of the highest occupied molecular orbital/band (HOMO), Fermi level, and the lowest unoccupied orbital/band (LUMO) of the amorphous LiGL bulk based on the structure changes in AIMD simulation for $15 \mathrm{ps}$ (Figure $8(\mathrm{~b})$ ). Our simulations revealed that, as shown in Figure 8(b), the electronic gap (or band gap) stays constant $\sim 3 \mathrm{eV}$ from $15 \mathrm{ps}$ AIMD simulation. In other words, this suggests that LiGL is insulating with $\sim 3 \mathrm{eV}$ in band gap at room temperature $(T \sim 300 \mathrm{~K})$ (Figure $8(\mathrm{~b})$ ), which is slightly smaller than the crystalline $\mathrm{Li}_{2} \mathrm{O}(\sim 4.3 \mathrm{eV})$ and $\mathrm{Li}_{2} \mathrm{CO}_{3}(\sim 4.5 \mathrm{eV})$ (Figure S17). Interestingly, this insulating merit can be persisted even at higher temperatures (e.g., $\sim 550 \mathrm{~K})$ with a slightly smaller band gap $\sim 2.2 \mathrm{eV}$ (Figure S18). Thus, we believe that the electronically insulating nature of the LiGL films must have helped suppress the chemically reactive metallic Li dendrites even at elevated temperatures. Our experimental measurements also verified the computational results and further revealed that the LiGL coating is ionically conductive. All these results will be reported in a following systematic study.
We further studied the evolution of cell impedance with cycles (Figure $8(c)$ ) to verify the protective effects of the LiGL coatings. Prior to Li-stripping/plating cycles, the uncycled bare $\mathrm{Li} / \mathrm{Li}$ cell has an impedance of $\sim 176 \Omega$ while the uncycled LiGL-60/LiGL-60 cell has an impedance of $\sim 312 \Omega$ due to the LiGL coatings (Figure 8(c)). However, after $1^{\text {st }}$ stripping/platting cycle, two semicircles were observed on the impedance profiles of both the bare $\mathrm{Li} / \mathrm{Li}$ and LiGL-60/LiGL-60 cells. The equivalent circuit is included as inset with the impedance profiles of the bare $\mathrm{Li} / \mathrm{Li}$ cell. $R_{b}$, $R_{\text {int }}$, and $R_{\mathrm{ct}}$ are the bulk resistor, the interfacial resistor, and charge transference resistor, respectively $[42,43]$. CPE represents a constant phase element. Apparently, the $R_{b}, R_{\text {int }}$, and $R_{\mathrm{ct}}$ of the LiGL-60/LiGL60 are much smaller than those of the bare Li/Li after the same cycles. This again confirmed that the LiGL coatings are very effective in stabilizing the Li interface and thereby reducing cell impedance during the Li-stripping/plating cycles.

3.5. The Proposed Mechanism of the LiGL Protection Effects on Li Electrodes. Based on the aforediscussed experimental and simulation results, we proposed the following mechanisms of Li-stripping/plating for the bare $\mathrm{Li} / \mathrm{Li}$ cell and the LiGL/LiGL cell, as illustrated in Figures 9(a) and 9(b), respectively.

As shown in Figure 9(a) for the evolution of the crosssection of a bare Li electrode, an Li chip is initially smooth (Figure 9(a1)) but becomes bumpy after the assembling press (Figure 9(a2)). At the same time, the Li chip surface has been formed with an SEI layer, due to its contact and reaction with the electrolyte (Figure 9(a2)). The surface bumps of the Li chip are prone to take the priority to start an Li-stripping earlier (Figure 9(a3)). With the depletion of the Li bumps, the surrounding areas become new bumps and take the priority to continue the stripping while the former bumps become craters covered a layer of SEI and some residuals of SEI from the depleted bumps (Figure 9(a4)). Thus, there are more SEI produced during the stripping, and the Li chip surface is not even in composition and morphology after the stripping (Figure 9(a5)). Due to the uneven surface property of the $\mathrm{Li}$ chip surface, the Li deposition in a subsequent plating is not uniform. Some areas of the Li chip surface are deposited with more $\mathrm{Li}$, and the deposited $\mathrm{Li}$ is prone to form dendritic structures (Figure 9(a6)). The formed Li dendritic structures further aggravate the uneven properties of the Li chip surface and exacerbate the formation of the SEI layer on the top of the Li chip in the following stripping (Figure 9(a7)). Some SEI residuals and dead Li may be isolated and dispersed in the electrolyte. After multiple cycles of such Li-stripping/plating process, the Li chip is significantly corroded and covered with a considerably thick SEI layer (Figure 9(a8)). At the same time, there is a significant consumption of Li and electrolyte. Eventually, the cell may be dried and have a significant increase of cell impedance and cell overpotential.

Different from the bare $\mathrm{Li}$ chip as illustrated in Figure 9(a), the LiGL MLD process can form a uniform coating over the Li chip surface (Figure 9(b1)). After the mechanical assembling press, the LiGL-coated Li chip becomes bumpy (Figure 9(b2)). During this press, the LiGL coating 


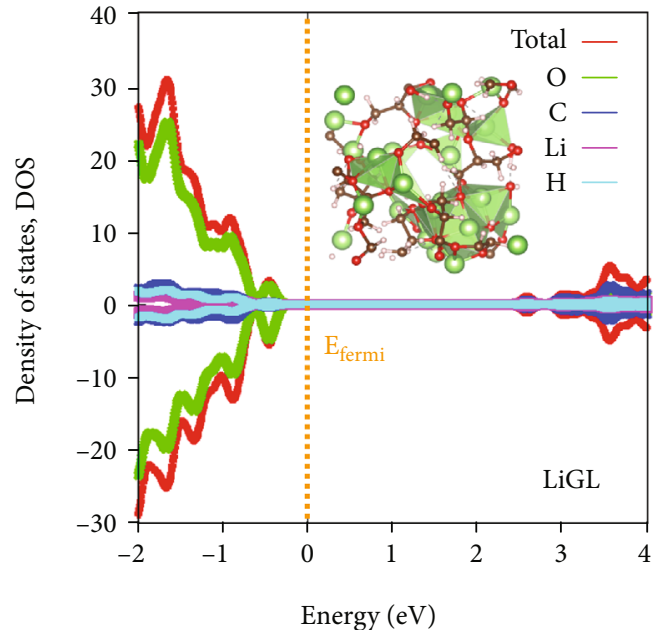

(a)

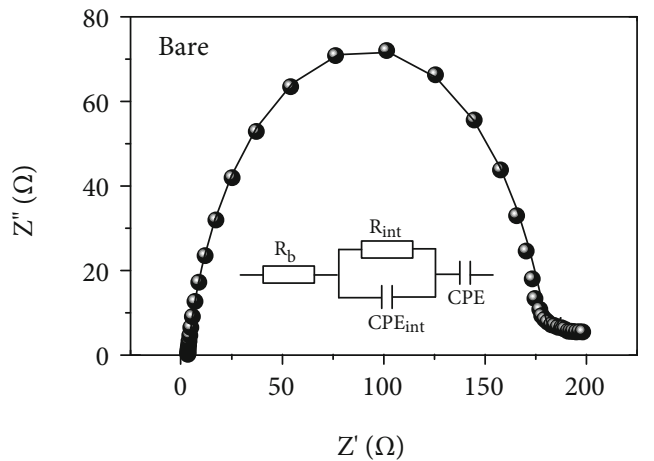

- Uncycled

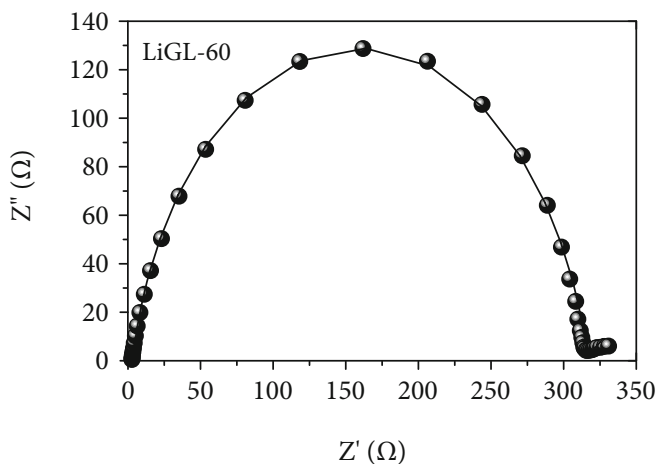

- Uncycled

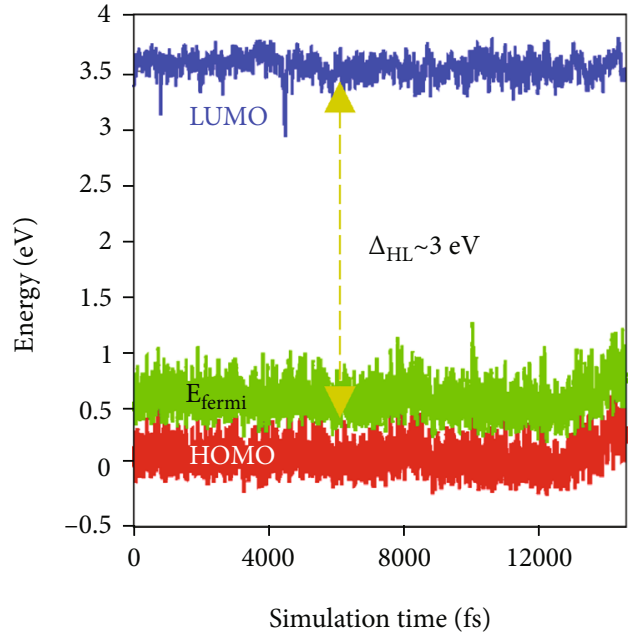

(b)

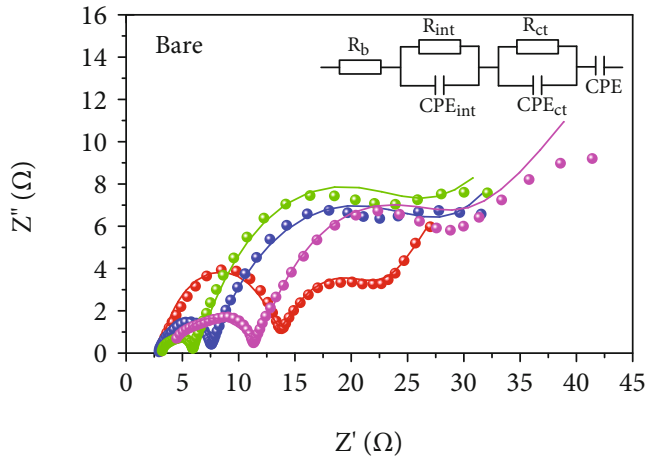
- 1 cycle;
- 5 cycles;
_ Simulation
- 20 cycles;
Simulation
- 50 cycles;
— Simulation

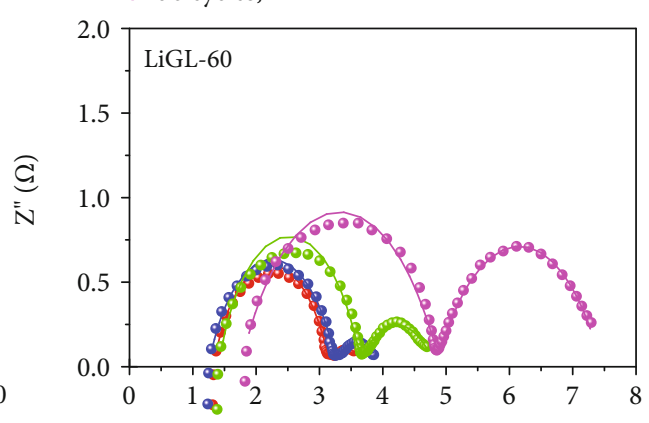

$Z^{\prime}(\Omega)$
- 1 cycle;
- 5 cycles;
- 20 cycles;
- Simulation
— Simulation
- 50 cycles;
_ Simulation
_ Simulation

(c)

FIGURE 8: Computational simulations on the electronic density of states (DOS) and on the time evolution of the highest occupied molecular orbital/band (HOMO), Fermi level, and the lowest unoccupied orbital/band (LUMO) of amorphous LiGL bulk. (a) DOS of LiGL bulk shows the insulating gap of $\sim 3 \mathrm{eV}$ (inset: amorphous LiGL bulk). (b) The time evolutions of HOMO, Fermi level, and LUMO show a consistent $\sim 3 \mathrm{eV}$ band gap from $15 \mathrm{ps}$ AIMD trajectories at room temperature $(T=300 \mathrm{~K})$. (c) The EIS measurements of the bare Li/Li and LiGL60/LiGL-60 cells before cycling and after different cycles, which have been cycled at $2 \mathrm{mAh} / \mathrm{cm}^{2}$. 


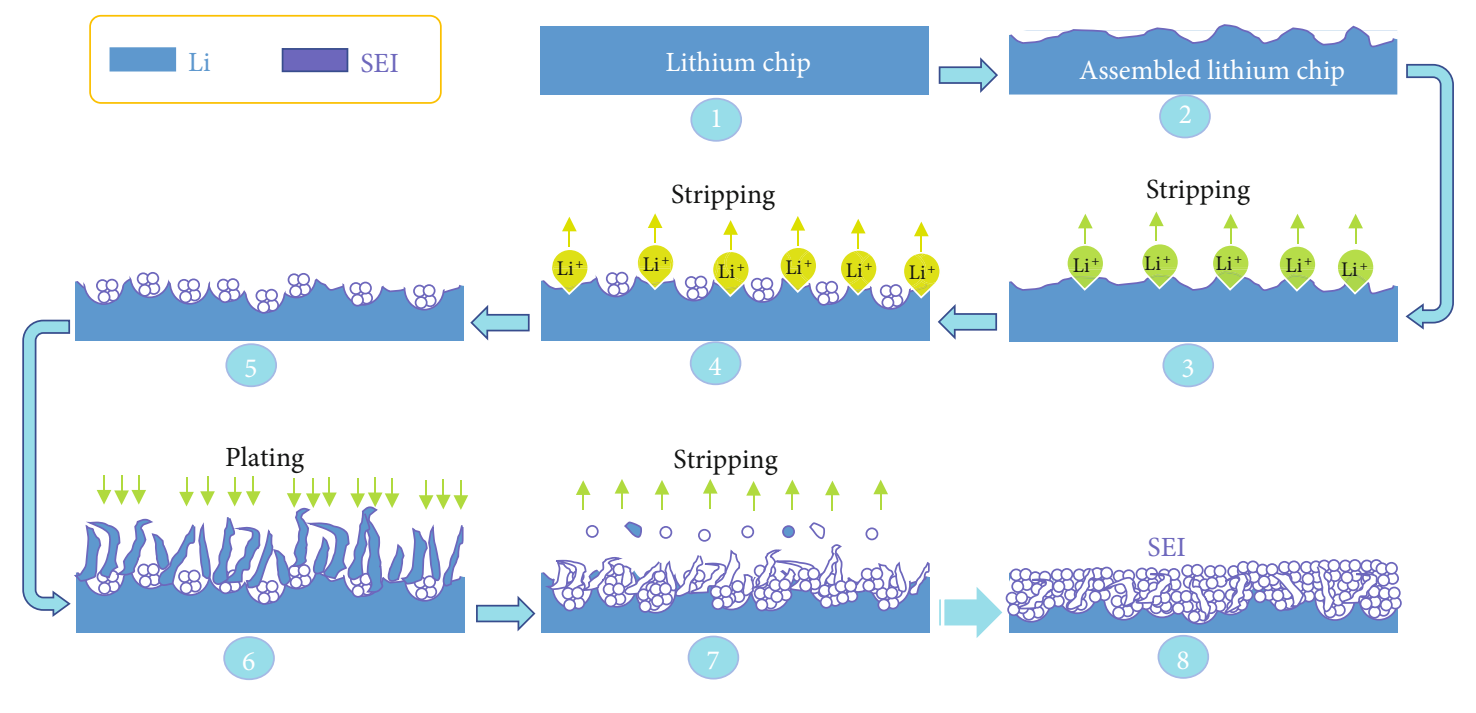

(a)

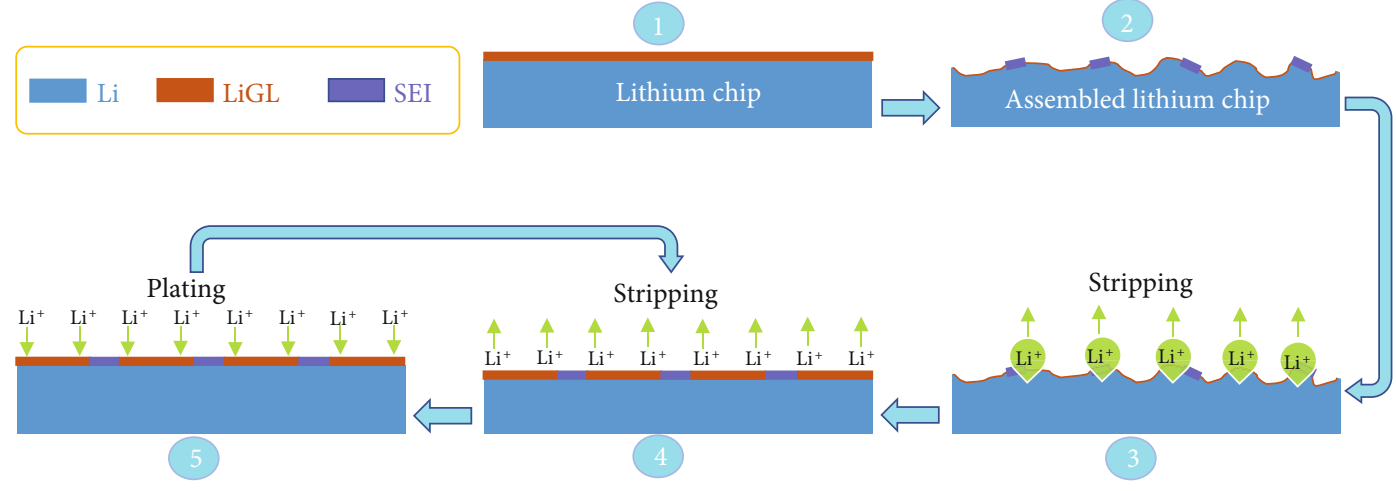

(b)

FIGURE 9: Schematic illustrations of the proposed mechanisms of Li-stripping/plating for bare $\mathrm{Li}$ and LiGL-coated Li. (a) the Li-stripping/plating mechanism of bare $\mathrm{Li}$ and (b) the Li-stripping/plating mechanism of LiGL-coated Li.

breaks into small pieces. The fractures contact the electrolyte and form an SEI layer. In comparison, the majority of the $\mathrm{Li}$ chip surface is still covered by the LiGL coating, and only a very small part of the surface is covered by an SEI layer. The LiGL coating is electronically insulating while ionically conductive. As a result, the stripping starts from the bumpy areas (Figure 9 (b3)) but the Li chip surface becomes even quickly with the depleted bumps (Figure 9 (b4)). Owing to the uniformity and the exceptional properties of the LiGL coating, the following stripping is remarkably even over the whole Li chip. Due to the uniform properties of the LiGLcoated Li chip surface, the following plating is also even without any further SEI formation (Figure 9(b5)). Consequently, the LiGL-coated Li chip can realize long-term stable cyclability without evident consumption of Li and electrolyte. Thus, the cell overpotential can sustain stable with extended cycles.

\section{Conclusion}

In summary, we for the first time developed a novel lithicone, LiGL, in this study, which can be deposited uniformly and conformally with an accurately controllable GPC at a moderate temperature of $150^{\circ} \mathrm{C}$. Significantly, this LiGL MLD has a decent average GPC of $\sim 2.7 \mathrm{~nm} /$ cycle and shows exceptional protection effects on Li electrodes, i.e., remarkably suppressing Li dendrites and mitigating SEI formation. Furthermore, we have clearly explored the protective mechanism of the LiGL coatings experimentally and computationally. Our computational simulations and experiments revealed that the MLD LiGL films are electrically insulating and ionically conductive. In addition, experimental results revealed a moderate stiffness of the LiGL coating. All these properties of the LiGL coatings underlie their excellent protective effects on $\mathrm{Li}$ metal electrodes. This work represents a facile solution to achieve high-performance Li metal anodes.

\section{Data Availability}

All data needed to evaluate the conclusions in the paper are present in the paper and the Supplementary Materials. Additional data related to this paper may be requested from the corresponding authors. 


\section{Conflicts of Interest}

The authors declare no conflict of interest.

\section{Authors' Contributions}

X.M. and K. C. L proposed the project, designed the experiments, and drafted and revised the manuscript. X. M. conducted electrochemical evaluations of cells, EIS measurements, SEM characterization, and MLD growth. K. C. L. performed computational calculations. H. Z. performed X-ray diffraction measurements. S. K. G. and M. Z. performed measurements of AFM. M. B. conducted some XPS characterizations.

\section{Acknowledgments}

X.M., M. Z., and S. K. G. acknowledge partial support from the Center for Advanced Surface Engineering, under the National Science Foundation Grant No. OIA-1457888 and the Arkansas EPSCoR Program, ASSET III. X. M. also appreciates the financial research support of the Chancellor's Fund from University of Arkansas, Fayetteville, AR, USA. K.C.L acknowledges the start-up fund provided by the California State University Northridge and financial support from Cottrell Scholar Award (Award\# 26829) by Research Corporation for Science Advancement (RCSA). This research used resources of the Advanced Photon Source, a U.S. Department of Energy (DOE) Office of Science User Facility, operated for the DOE Office of Science by Argonne National Laboratory under Contract No. DE-AC02-06CH11357. Extraordinary facility operations were supported in part by the DOE Office of Science through the National Virtual Biotechnology Laboratory, a consortium of DOE national laboratories focused on the response to COVID-19, with funding provided by the Coronavirus CARES Act. X. M. appreciates the assistance of Dr. Fumiya Watanabe in materials characterization using XPS at Center for Integrative Nanotechnology Sciences at University of Arkansas at Little Rock (Little Rock, AR).

\section{Supplementary Materials}

Figure S1. Comparison of the Li-stripping/plating cyclability of Li metal electrodes coated by our LiGL in this work and by other MLD films and other promising coatings. Figure S2. Growth of LiEG and LiHQ lithicones via MLD. (a) QCM measurements of MLD LiEG at $150{ }^{\circ} \mathrm{C}$ and (b) repeatable MLD cycles in stable growth region. (c) QCM measurements of MLD LiHQ at $150{ }^{\circ} \mathrm{C}$ and (d) repeatable MLD cycles in stable growth region. Figure S3. Growth of LiGL via MLD. QCM measurements of MLD LiEG in 100 cycles at $150{ }^{\circ} \mathrm{C}$, which can be divided into two regions: the initiation and stable growth region. Table S1. Elements and Quantifications of MLD LiGL Films. Figure S4. XPS depth profiling of a 75MLD-cycle LiGL film. The LiGL film can be divided into four parts: (I) Li-deficient layer, (II) stoichiometrically stable layer, (III) Li-rich layer, and (IV) interface layer. Table S2. Elements and Quantifications of LiGL films exposed in air for $1 \mathrm{~h}$. Figure S5. XPS analyses on (a) pristine LiGL films and (b) 1-h air-exposed LiGL films. Figure S6. XRD analyses on GL, N-GNS, and 75-MLD-cycle LiGL-coated N-GNS. The peak at $17^{\circ}$ is from the background. Figure S7. Densityfunctional theory (DFT) simulations on amorphous LiGL films. (a) The RDF of $\mathrm{Li}-\mathrm{O}$ bonds from amorphous $\mathrm{LiGL}$ and crystalline $\mathrm{Li}_{2} \mathrm{O}$ obtained from DFT results. (b) The $\mathrm{RDF}$ of $\mathrm{C}-\mathrm{O}, \mathrm{C}-\mathrm{H}$, and $\mathrm{C}-\mathrm{D}$ bonds found in amorphous $\mathrm{LiGL}$ bulk at $\mathrm{T}=300 \mathrm{~K}$ obtained from AIMD. Figure S8. Evolutions of overpotentials of LiGL-20, LiGL-60, and LiGL-90 electrodes with cycle number at a current density of $2 \mathrm{~mA} / \mathrm{cm}^{2}$ and an areal capacity of $1 \mathrm{mAh} / \mathrm{cm}^{2}$. The overpotential profiles are compared for three $(A, B$, and $C)$ segments of 50 cycles. Figure S9. Evolutions of overpotentials of LiGL20 and LiGL-60 electrodes with cycle number at a current density of $5 \mathrm{~mA} / \mathrm{cm}^{2}$ and an areal capacity of $1 \mathrm{mAh} / \mathrm{cm}^{2}$. The overpotential profiles are compared for 4 (A, B, C, and D) segments of 50 cycles. Figure S10. Electrochemical evaluations of LiGL-coated $\mathrm{Li}$ electrodes in $\mathrm{Li} / \mathrm{Li}$ symmetric cells. $\mathrm{Li} / \mathrm{Li}$ cells tested at $2 \mathrm{~mA} / \mathrm{cm}^{2}$ for the first 200 cycles and at $7.5 \mathrm{~mA} / \mathrm{cm}^{2}$ for the subsequent cycles with a fixed areal capacity of $1 \mathrm{mAh} / \mathrm{cm}^{2}$. Figure S11. Surface morphology of the cycled LiGL-60 electrode after $700 \mathrm{Li}$-stripping/plating. The LiGL-60/LiGL-60 cell was cycled at $2 \mathrm{~mA} / \mathrm{cm}^{2}$ and at a fixed areal capacity of $1 \mathrm{mAh} / \mathrm{cm}^{2}$. Figure $\mathrm{S} 12$. The SEM image of the cross section of the bare Li electrode before cycling. Figure S13. XPS analyses of the bare Li and LiGL90 electrodes. High-resolution XPS spectra of (a) the bare $\mathrm{Li}$ and (b) LiGL-90 electrode after $10 \mathrm{Li}$-stripping/plating cycles at $2 \mathrm{~mA} / \mathrm{cm}^{2}$ and $1 \mathrm{mAh} / \mathrm{cm}^{2}$. Figure S14. SEM observations of the morphological changes of Li electrodes. $(a, b)$ the bare $\mathrm{Li}$ and $(\mathrm{c}, \mathrm{d}) \mathrm{LiGL}-20$ electrode after one stripping (or plating) at a current density of $2 \mathrm{~mA} / \mathrm{cm}^{2}$ and an areal capacity of $1 \mathrm{mAh} / \mathrm{cm}^{2}$. Figure S15. SEM observations of the morphological changes of Li electrodes after the press of an assembling. (a) Li chip before assembling (b-f) Li chip after assembling. Figure S16. SEM observations of the morphological changes of LiGL electrodes after the press of an assembling. (a) LiGL-coated Li chip before assembling and (b-d) LiGL-coated Li chip after assembling. Figure S17. DFT simulations on the electronic density of states (DOS) of $\mathrm{Li}_{2} \mathrm{CO}_{3}$ and $\mathrm{Li}_{2} \mathrm{O}$. DOS of (a) crystalline $\mathrm{Li}_{2} \mathrm{CO}_{3}$ and (b) crystalline $\mathrm{Li}_{2} \mathrm{O}$ shows finite band gap of $\sim 4.5$ and $\sim 4.3 \mathrm{eV}$, respectively. Figure S18. The time evolution of the highest occupied molecular orbital/band (HOMO), fermi level, and the lowest unoccupied orbital/band (LUMO) of LiGL films. Results show that a smaller band gap $\sim 2.2 \mathrm{eV}$ from $15 \mathrm{ps}$ AIMD trajectories at (a) $\mathrm{T}=400 \mathrm{~K}$ (b) $\mathrm{T}=550 \mathrm{~K}$. (Supplementary Materials)

\section{References}

[1] W. Xu, J. Wang, F. Ding et al., "Lithium metal anodes for rechargeable batteries," Energy \& Environmental Science, vol. 7, no. 2, pp. 513-537, 2014.

[2] M. S. Whittingham, "Electrical energy storage and intercalation chemistry," Science, vol. 192, no. 4244, pp. 1126-1127, 1976.

[3] S. Ni, S. Tan, Q. An, and L. Mai, "Three dimensional porous frameworks for lithium dendrite suppression," Journal of Energy Chemistry, vol. 44, pp. 73-89, 2020.

[4] H. Zhang, G. G. Eshetu, X. Judez, C. Li, L. M. Rodriguez-Martínez, and M. Armand, "Electrolyte additives for Lithium metal 
anodes and rechargeable lithium metal batteries: progress and perspectives," Angewandte Chemie, International Edition, vol. 57, no. 46, pp. 15002-15027, 2018.

[5] T. Zhang, W. He, W. Zhang et al., "Designing composite solidstate electrolytes for high performance lithium ion or lithium metal batteries," Chemical Science, vol. 11, no. 33, pp. 86868707, 2020.

[6] Q. Sun, K. C. Lau, D. Geng, and X. Meng, “Atomic and molecular layer deposition for superior lithium-sulfur batteries: strategies, performance, and mechanisms," Batteries \& Supercaps, vol. 1, no. 2, pp. 41-68, 2018.

[7] X. Meng, "Atomic layer deposition of solid-state electrolytes for next-generation lithium-ion batteries and beyond: opportunities and challenges," Energy Storage Materials, vol. 30, pp. 296-328, 2020.

[8] H. Zhou, S. Yu, H. Liu, and P. Liu, "Protective coatings for lithium metal anodes: recent progress and future perspectives," Journal of Power Sources, vol. 450, p. 227632, 2020.

[9] Q. Yang, W. Li, C. Dong et al., "PIM-1 as an artificial solid electrolyte interphase for stable lithium metal anode in highperformance batteries," Journal of Energy Chemistry, vol. 42, pp. 83-90, 2020.

[10] R. Xu, X.-B. Cheng, C. Yan et al., “Artificial interphases for highly stable Lithium metal anode," Matter, vol. 1, no. 2, pp. 317-344, 2019.

[11] Y. Sun, M. Amirmaleki, Y. Zhao et al., "Tailoring the mechanical and electrochemical properties of an artificial interphase for high-performance metallic lithium anode," Advanced Energy Materials, vol. 10, no. 28, p. 2001139, 2020.

[12] X. Meng, "An overview of molecular layer deposition for organic and organic-inorganic hybrid materials: mechanisms, growth characteristics, and promising applications," Journal of Materials Chemistry A, vol. 5, no. 35, pp. 18326-18378, 2017.

[13] X. Meng, "Atomic and molecular layer deposition in pursuing better batteries," Journal of Materials Research, vol. 36, no. 1, pp. 2-25, 2021.

[14] Y. Sun, Y. Zhao, J. Wang et al., "A novel organic "Polyurea" thin film for ultralong-life lithium-metal anodes via molecular-layer deposition," Advanced Materials, vol. 31, no. 4, p. $1806541,2019$.

[15] L. Chen, Z. Huang, R. Shahbazian-Yassar et al., "Directly formed alucone on lithium metal for high-performance Li batteries and Li-S batteries with high sulfur mass loading," ACS Applied Materials \& Interfaces, vol. 10, no. 8, pp. 7043-7051, 2018.

[16] Y. Zhao, L. V. Goncharova, Q. Sun et al., "Robust metallic lithium anode protection by the molecular-layer-deposition technique," Small Methods, vol. 2, no. 5, p. 1700417, 2018.

[17] K. R. Adair, C. Zhao, M. N. Banis et al., "Highly stable lithium metal anode interface via molecular layer deposition zircone coatings for long life next-generation battery systems," Angewandte Chemie, International Edition, vol. 58, no. 44, pp. 15797-15802, 2019.

[18] J. P. Perdew, K. Burke, and M. Ernzerhof, "Generalized gradient approximation made simple," Physical Review Letters, vol. 77, no. 18, pp. 3865-3868, 1996.

[19] P. E. Blöchl, "Projector augmented-wave method," Physical Review B, vol. 50, no. 24, pp. 17953-17979, 1994

[20] S. Grimme, "Semiempirical GGA-type density functional constructed with a long-range dispersion correction," Journal of
Computational Chemistry, vol. 27, no. 15, pp. 1787-1799, 2006.

[21] G. Kresse and J. Furthmüller, "Efficiency of ab-initio total energy calculations for metals and semiconductors using a plane-wave basis set," Computational Materials Science, vol. 6, no. 1, pp. 15-50, 1996.

[22] G. Kresse and D. Joubert, "From ultrasoft pseudopotentials to the projector augmented-wave method," Physical Review B, vol. 59, no. 3, pp. 1758-1775, 1999.

[23] Y. Cao, X. Meng, and J. W. Elam, “Atomic layer deposition of $\mathrm{Li}_{\mathrm{x}} \mathrm{Al}_{\mathrm{y}} \mathrm{S}$ solid-state electrolytes for stabilizing lithium-metal anodes," ChemElectroChem, vol. 3, no. 6, pp. 858-863, 2016.

[24] X. Wang, S. K. Ghosh, M. Afshar-Mohajer et al., "Atomic layer deposition of zirconium oxide thin films," Journal of Materials Research, vol. 35, no. 7, pp. 804-812, 2019.

[25] J. Cai, Z. Ma, U. Wejinya et al., "A revisit to atomic layer deposition of zinc oxide using diethylzinc and water as precursors," Journal of Materials Science, vol. 54, no. 7, pp. 5236-5248, 2019.

[26] S. M. George, B. H. Lee, B. Yoon, A. I. Abdulagatov, and R. A. Hall, "Metalcones: hybrid organic-inorganic films fabricated using atomic and molecular layer deposition techniques," Journal of Nanoscience and Nanotechnology, vol. 11, no. 9, pp. 7948-7955, 2011.

[27] J. Cai, B. Reinhart, P. Eng et al., "Nitrogen-doped graphenewrapped $\mathrm{Cu}_{2} \mathrm{~S}$ as a superior anode in sodium-ion batteries," Carbon, vol. 170, pp. 430-438, 2020.

[28] A. Liu, G. Liu, C. Zhu et al., "Solution-processed alkaline lithium oxide dielectrics for applications in n- and p-type thin-film transistors," Advanced Electronic Materials, vol. 2, no. 9, p. 1600140, 2016.

[29] K. N. Wood and G. Teeter, "XPS on Li-battery-related compounds: analysis of inorganic SEI phases and a methodology for charge correction," ACS Applied Energy Materials, vol. 1, no. 9, pp. 4493-4504, 2018.

[30] L. J. Rendek, G. S. Chottiner, and D. A. Scherson, "The reactivity of linear alkyl carbonates toward metallic lithium," Journal of the Electrochemical Society, vol. 149, no. 10, article E408, 2002.

[31] H. Wang, K. E. Gregorczyk, S. B. Lee, G. W. Rubloff, and C.-F. Lin, "Li-containing organic thin film-structure of lithium propane dioxide via molecular layer deposition," Journal of Physical Chemistry C, vol. 124, no. 12, pp. 6830-6837, 2020.

[32] D. Bar-Tow, E. Peled, and L. Burstein, "A study of highly oriented pyrolytic graphite as a model for the graphite anode in Li-ion batteries," Journal of the Electrochemical Society, vol. 146, no. 3, pp. 824-832, 1999.

[33] A. C. W. Tan, B. J. Polo-Cambronell, E. Provaggi et al., "Design and development of low cost polyurethane biopolymer based on castor oil and glycerol for biomedical applications," Biopolymers, vol. 109, no. 2, article e23078, 2018.

[34] J. Zheng, P. Yan, D. Mei et al., "Highly stable operation of lithium metal batteries enabled by the formation of a transient high-concentration electrolyte layer," Advanced Energy Materials, vol. 6, no. 8, p. 1502151, 2016.

[35] X. Zhou, Y. Zhang, Z. Huang, D. Lu, A. Zhu, and G. Shi, "Ionic liquids modified graphene oxide composites: a high efficient adsorbent for phthalates from aqueous solution," Scientific Reports, vol. 6, no. 1, p. 38417, 2016.

[36] K. Kanamura, H. Tamura, S. Shiraishi, and Z.-I. Takehara, "XPS analysis for the lithium surface immersed in $\gamma$ - 
butyrolactone containing various salts," Electrochimica Acta, vol. 40, no. 7, pp. 913-921, 1995.

[37] X. Meng, Y. Cao, J. A. Libera, and J. W. Elam, “Atomic layer deposition of aluminum sulfide: growth mechanism and electrochemical evaluation in lithium-ion batteries," Chemistry of Materials, vol. 29, no. 21, pp. 9043-9052, 2017.

[38] D. Seghete, R. A. Hall, B. Yoon, and S. M. George, "Importance of trimethylaluminum diffusion in three-step ABC molecular layer deposition using trimethylaluminum, ethanolamine, and maleic anhydride," Langmuir, vol. 26, no. 24, pp. 1904519051, 2010.

[39] Q. Ma, J. Yue, M. Fan et al., "Formulating the electrolyte towards high-energy and safe rechargeable lithium-metal batteries," Angewandte Chemie, International Edition, vol. 60, no. 30, pp. 16554-16560, 2021.

[40] J. Liu, Z. Bao, Y. Cui et al., "Pathways for practical high-energy long-cycling lithium metal batteries," Nature Energy, vol. 4, no. 3, pp. 180-186, 2019.

[41] A. Masias, N. Felten, R. Garcia-Mendez, J. Wolfenstine, and J. Sakamoto, "Elastic, plastic, and creep mechanical properties of lithium metal," Journal of Materials Science, vol. 54, no. 3, pp. 2585-2600, 2019.

[42] K. Wen, L. Liu, S. Chen, and S. Zhang, "A bidirectional growth mechanism for a stable lithium anode by a platinum nanolayer sputtered on a polypropylene separator," RSC Advances, vol. 8, no. 23, pp. 13034-13039, 2018.

[43] C. He, J. Liu, J. Cui, J. Li, and X. Wu, “A gel polymer electrolyte based on Polyacrylonitrile/organic montmorillonite membrane exhibiting dense structure for lithium ion battery," Solid State Ionics, vol. 315, pp. 102-110, 2018. 